\title{
ORBITS OF DISCRETE SUBGROUPS ON A SYMMETRIC SPACE AND THE FURSTENBERG BOUNDARY
}

\section{ALEXANDER GORODNIK and HEE OH}

\begin{abstract}
Let $X$ be a symmetric space of noncompact type, and let $\Gamma$ be a lattice in the isometry group of $X$. We study the distribution of orbits of $\Gamma$ acting on the symmetric space $X$ and its geometric boundary $X(\infty)$, generalizing the main equidistribution result of Margulis's thesis [M, Theorem 6] to higher-rank symmetric spaces. More precisely, for any $y \in X$ and $b \in X(\infty)$, we investigate the distribution of the set $\left\{\left(y \gamma, b \gamma^{-1}\right)\right.$ : $\gamma \in \Gamma\}$ in $X \times X(\infty)$. It is proved, in particular, that the orbits of $\Gamma$ in the Furstenberg boundary are equidistributed and that the orbits of $\Gamma$ in $X$ are equidistributed in "sectors" defined with respect to a Cartan decomposition. Our main tools are the strong wavefront lemma and the equidistribution of solvable flows on homogeneous spaces, which we obtain using Shah's result [S, Corollary 1.2] based on Ratner's measure-classification theorem [R1, Theorem 1].
\end{abstract}

\section{Contents}

1. Introduction . . . . . . . . . . . . . . . . . . . . 484

2. Main ingredients of the proofs . . . . . . . . . . . . . . . 489

3. Cartan decomposition and the strong wavefront lemma . . . . . . . . 493

4. Contractions on $G / B \ldots \ldots$. . . . . . . . . . . . . . . . . . 499

5. Volume estimates . . . . . . . . . . . . . . . . . . . 502

6. Equidistribution of solvable flows . . . . . . . . . . . 506

7. Distribution of lattice points in sectors and the boundary . . . . . . . . 513

8. Distribution of lattice points in bisectors . . . . . . . . . . . . 518

9. Measure-preserving lattice actions . . . . . . . . . . . . . . . . 522

References ....................... 523

DUKE MATHEMATICAL JOURNAL

Vol. 139, No. 3, (c) 2007

Received 6 September 2006. Revision received 12 December 2006.

2000 Mathematics Subject Classification. Primary 22E40; Secondary 37A17.

Gorodnik's work partially supported by National Science Foundation grant DMS-0400631.

Oh's work partially supported by National Science Foundation grant DMS-0333397. 


\section{Introduction}

Let $\mathbb{D}$ denote the hyperbolic unit disc, and let $\Gamma$ denote a torsion-free discrete subgroup of the isometry group of $\mathbb{D}$ such that $\mathbb{D} / \Gamma$ has finite area. The geometric boundary of $\mathbb{D}$ is the space of the equivalence classes of geodesic rays in $\mathbb{D}$. It can be identified with the unit circle $\mathbb{S}$. Note that the action of $\Gamma$ on $\mathbb{D}$ extends to the geometric boundary of $\mathbb{D}$.

Let $x \in \mathbb{D}$. We denote by $\mathrm{B}_{\mathrm{T}}(x)$ the ball of radius $T$ centered at $x$. For an arc $\Omega \subset \mathbb{S}$, the sector $\mathscr{S}_{x}(\Omega)$ in $\mathbb{D}$ is defined to be the set of points $z \in \mathbb{D}$ such that the end point of the geodesic ray from $x$ to $z$ lies in $\Omega$. Denote by $m_{x}$ the unique probability measure on $\mathbb{S}$ invariant under the isometries that fix the point $x$. Then:

(A) for any $x, y \in \mathbb{D}, b \in \mathbb{S}$, and an $\operatorname{arc} \Omega \subset \mathbb{S}$,

$$
\#\left\{\gamma \in \Gamma: b \gamma^{-1} \in \Omega, y \gamma \in \mathrm{B}_{\mathrm{T}}(x)\right\} \sim_{T \rightarrow \infty} m_{y}(\Omega) \cdot \frac{\operatorname{Area}\left(\mathrm{B}_{\mathrm{T}}(x)\right)}{\operatorname{Area}(\mathbb{D} / \Gamma)} ;
$$

(B) for any $x, y \in \mathbb{D}$, and an $\operatorname{arc} \Omega \subset \mathbb{S}$,

$$
\#\left\{\gamma \in \Gamma: y \gamma \in \mathscr{S}_{x}(\Omega) \cap \mathrm{B}_{\mathrm{T}}(x)\right\} \sim_{T \rightarrow \infty} m_{x}(\Omega) \cdot \frac{\operatorname{Area}\left(\mathrm{B}_{\mathrm{T}}(x)\right)}{\operatorname{Area}(\mathbb{D} / \Gamma)} ;
$$

(C) for every $x, y \in \mathbb{D}, b \in \mathbb{S}$, and $\operatorname{arcs} \Omega_{1}, \Omega_{2} \subset \mathbb{S}$,

$$
\begin{gathered}
\#\left\{\gamma \in \Gamma: y \gamma \in \mathscr{S}_{x}\left(\Omega_{1}\right) \cap \mathrm{B}_{\mathrm{T}}(x), b \gamma^{-1} \in \Omega_{2}\right\} \\
\sim_{T \rightarrow \infty} m_{x}\left(\Omega_{1}\right) m_{y}\left(\Omega_{2}\right) \cdot \frac{\operatorname{Area}\left(\mathrm{B}_{\mathrm{T}}(x)\right)}{\operatorname{Area}(\mathbb{D} / \Gamma)}
\end{gathered}
$$

(see Figure 1).

Statement (A) may be deduced from the work of Good [G]. Statement (B) was shown by Nicholls [N] (see also [Sh]). Statement (C), which was proved by Margulis $[\mathrm{M}]$ for cocompact $\Gamma$, shows that the equidistribution phenomena in $(\mathrm{A})$ and $(\mathrm{B})$ are indeed independent.

The main purpose of this article is to obtain an analog of statement (C) (note that (C) implies both (A) and (B)) for an arbitrary Riemannian symmetric space of noncompact type (see Theorems 1.1, 1.2). We also generalize statement (B) to the equidistribution of lattice points in a connected noncompact semisimple Lie group $G$ with finite center with respect to both $K$-components in a Cartan decomposition $G=K A^{+} K$ (see Theorem 1.6).

Let $X$ be a Riemannian symmetric space of noncompact type, and let $X(\infty)$ be the geometric boundary of $X$ (i.e., the space of equivalence classes of geodesic rays in $X$ ). Denote by $G$ the identity component of the isometry group of $X$ acting on $X$ from the right-hand side. Let $\Gamma$ be a lattice in $G$ (i.e., a discrete subgroup with finite covolume). The action of $G$ on $X$ extends to $X(\infty)$. 


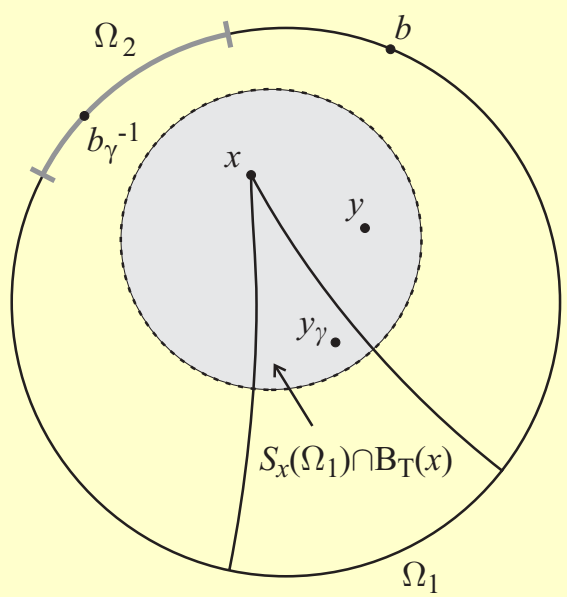

Figure 1

For $x \in X$, we denote by $\mathrm{B}_{\mathrm{T}}(x)$ the Riemannian ball of radius $T$ centered at $x$; by $K_{x}$ the stabilizer of $x$ in $G$; and by $\nu_{x}$ the probability Haar measure on $K_{x}$. For $x \in X$ and $b \in X(\infty)$, we denote by $m_{b, x}$ the unique probability $K_{x}$-invariant measure supported on the orbit $b G \subset X(\infty)$. (Note that $G$ acts transitively on $X(\infty)$ only when the rank of $X$ is one and that $K_{x}$ acts transitively on each $G$-orbit in $X(\infty)$.) Fix a closed Weyl chamber $\mathscr{W}_{x} \subset X$ at $x$. According to the Cartan decomposition, we have $X=\mathscr{W}_{x} K_{x}$. Let $M_{x}$ denote the stabilizer of $\mathscr{W}_{x}$ in $K_{x}$.

The following is one of our main theorems.

THEOREM 1.1

For $x, y \in X, b \in X(\infty)$, and any Borel subsets $\Omega_{1} \subset K_{x}$ and $\Omega_{2} \subset b G$ with boundaries of measure zero,

$\#\left\{\gamma \in \Gamma: y \gamma \in \mathscr{W}_{x} \Omega_{1} \cap \mathrm{B}_{\mathrm{T}}(x), b \gamma^{-1} \in \Omega_{2}\right\} \sim_{T \rightarrow \infty} v_{x}\left(M_{x} \Omega_{1}\right) m_{b, y}\left(\Omega_{2}\right) \cdot \frac{\operatorname{Vol}\left(\mathrm{B}_{\mathrm{T}}\right)}{\operatorname{Vol}(G / \Gamma)}$,

where $\operatorname{Vol}\left(\mathrm{B}_{\mathrm{T}}\right)$ denotes the volume of a ball of radius $T$ in $X$.

We deduce Theorem 1.1 from a stronger result on the level of Lie groups. Fix the following data:

- $G$ is a connected noncompact semisimple Lie group with finite center;

- $G_{n}$ is the product of all noncompact simple factors of $G$;

- $G=K_{1} A^{+} K_{1}$ is a Cartan decomposition of $G$;

- $d$ is an invariant metric on $K_{1} \backslash G$;

- $\quad K_{2}$ is a maximal compact subgroup of $G$; and

- $Q$ is a closed subgroup of $G$ which contains a maximal connected split solvable subgroup. 
Recall that a solvable subgroup $S$ is called split if the eigenvalues of any element of $\operatorname{Ad}(S)$ are real for the adjoint representation $\operatorname{Ad}: G \rightarrow \operatorname{GL}(\operatorname{Lie}(G))$. It is well known that a maximal connected split solvable subgroup is a subgroup of the form $A N$ for an Iwasawa decomposition $G=K A N$. Thus, $G=K_{2} Q$. Denote by $v_{1}$ and $\nu_{2}$ the probability Haar measure on $K_{1}$ and $K_{2}$, respectively. Let $M_{1}$ be the centralizer of $A$ in $K_{1}$, and let $M_{2}=K_{2} \cap Q$. Since any two maximal compact subgroups of $G$ are conjugate to each other, there exists $g \in G$ such that $K_{2}=g^{-1} K_{1} g$. Let $\Gamma$ be a lattice in $G$ such that $\overline{\Gamma G_{n} Q}=G$.

\section{THEOREM 1.2}

For any Borel subsets $\Omega_{1} \subset K_{1}$ and $\Omega_{2} \subset K_{2}$ with boundaries of measure zero,

$$
\begin{gathered}
\#\left\{\gamma \in \Gamma \cap g^{-1} K_{1} A^{+} \Omega_{1} \cap \Omega_{2} Q: d\left(K_{1}, K_{1} g \gamma\right)<T\right\} \\
\sim_{T \rightarrow \infty} v_{1}\left(M_{1} \Omega_{1}\right) \nu_{2}\left(\Omega_{2} M_{2}\right) \cdot \frac{\operatorname{Vol}\left(G_{T}\right)}{\operatorname{Vol}(G / \Gamma)},
\end{gathered}
$$

where $\operatorname{Vol}\left(G_{T}\right)$ denotes the volume of a Riemannian ball of radius $T$ in $G$.

To understand the presence of $M_{1}$ and $M_{2}$ in the above asymptotics, observe that $K_{1} A^{+} \Omega_{1}=K_{1} A^{+} M_{1} \Omega_{1}$ and $\Omega_{2} M_{2} Q=\Omega_{2} Q$.

\section{Remark 1.3}

We mention that the continuous version of Theorem 1.2 does not seem obvious, either. The method of the proof of Theorem 1.2 also yields the following volume asymptotics:

$$
\begin{gathered}
\operatorname{Vol}\left(\left\{h \in g^{-1} K_{1} A^{+} \Omega_{1} \cap \Omega_{2} Q: d\left(K_{1}, K_{1} g h\right)<T\right\}\right) \\
\sim_{T \rightarrow \infty} v_{1}\left(M_{1} \Omega_{1}\right) v_{2}\left(\Omega_{2} M_{2}\right) \cdot \frac{\operatorname{Vol}\left(G_{T}\right)}{\operatorname{Vol}(G / \Gamma)} .
\end{gathered}
$$

\section{Remark 1.4}

In Theorem 1.2, if we replace $K_{1} A^{+} \Omega_{1}$ by $\Omega_{1} A^{+} K_{1}$, then the statement of the theorem is false. In fact, we can show that there exist nonempty open subsets $\Omega_{1} \subset K_{1}$ and $\Omega_{2} \subset K_{2}$ such that

$$
\lim _{T \rightarrow \infty} \frac{1}{\operatorname{Vol}\left(G_{T}\right)} \operatorname{Vol}\left(\left\{h \in g^{-1} \Omega_{1} A^{+} K_{1} \cap \Omega_{2} Q: d\left(K_{1}, K_{1} g h\right)<T\right\}\right)=0 .
$$

To offer yet another generalization of statement (B), we fix a Cartan decomposition $G=K A^{+} K$ and an invariant Riemannian metric $d$ on $K \backslash G$. Let $\Gamma$ be any lattice in 
$G$. Recall that it was shown in [DRS] and [EM] that for a lattice $\Gamma$ in $G$,

$$
\#\{\gamma \in \Gamma: d(K, K g \gamma)<T\}=\#\left(\Gamma \cap g^{-1} K A_{T}^{+} K\right) \sim_{T \rightarrow \infty} \frac{\operatorname{Vol}\left(G_{T}\right)}{\operatorname{Vol}(G / \Gamma)},
$$

where $A_{T}^{+}=\left\{a \in A^{+}: d(K, K a)<T\right\}$. The following theorem is a generalization of this result.

\section{THEOREM 1.6}

For $g \in G$ and any Borel subsets $\Omega_{1} \subset K$ and $\Omega_{2} \subset K$ with boundaries of measure zero,

$\#\left(\Gamma \cap g^{-1} \Omega_{1} A_{T}^{+} M \Omega_{2}\right) \sim_{T \rightarrow \infty} \frac{\operatorname{Vol}\left(g^{-1} \Omega_{1} A_{T}^{+} M \Omega_{2}\right)}{\operatorname{Vol}(G / \Gamma)}=v\left(\Omega_{1} M\right) v\left(M \Omega_{2}\right) \cdot \frac{\operatorname{Vol}\left(G_{T}\right)}{\operatorname{Vol}(G / \Gamma)}$,

where $M$ is the centralizer of $A^{+}$in $K$ and $v$ is the probability Haar measure on $K$.

We now present several corollaries of the methods of Theorems 1.1, 1.2, and 1.6.

\subsection{Lattice action on the Furstenberg boundary}

For a connected semisimple Lie group $G$ with finite center, the Furstenberg boundary of $G$ is identified with the quotient space $G / P$, where $P$ is a minimal parabolic subgroup of $G$ (see [GJT, Chapter IV]). In the rank-one case, the Furstenberg boundary $G / P$ coincides with the geometric boundary $X(\infty)$ of the symmetric space $X$ of $G$. In the higher-rank case, $G / P$ is isomorphic to the $G$-orbit in $X(\infty)$ of any regular geodesic class and can be identified with the space of asymptotic classes of Weyl chambers in $X$.

It is well known that the action of a lattice $\Gamma$ on $G / P$ is minimal; that is, every $\Gamma$-orbit is dense (see [Mo, Lemma 8.5]). A natural question is whether each $\Gamma$-orbit in $G / P$ is equidistributed. Corollary 1.7 , which is a special case of Theorem 1.2 , implies an affirmative answer in a much more general setting.

Let $d$ denote an invariant Riemannian metric on the symmetric space $X \simeq K \backslash G$, where $K$ is a maximal compact subgroup of $G$.

COROLLARY 1.7

Let $Q$ be a closed subgroup of $G$ containing a maximal connected split solvable subgroup of $G$, and let $g \in G$. Denote by $v_{g}$ the unique $g^{-1} \mathrm{Kg}$-invariant probability measure on $G / Q$. Let $b \in G / Q$, and let $\Gamma$ be a lattice in $G$ such that $\overline{\Gamma G_{n} b}=G / Q$. Then for any Borel subset $\Omega \subset G / Q$ such that $\nu_{g}(\partial \Omega)=0$,

$$
\#\{\gamma \in \Gamma: \gamma b \in \Omega, d(K, K g \gamma)<T\} \sim_{T \rightarrow \infty} v_{g}(\Omega) \cdot \frac{\operatorname{Vol}\left(G_{T}\right)}{\operatorname{Vol}(G / \Gamma)} .
$$


It follows from Shah's result [S, Theorem 1.1] and Ratner's topological rigidity theorem [R2, Theorem 4] that the condition $\overline{\Gamma G_{n} b}=G / Q$ is equivalent to the density of the orbit $\Gamma b$ in $G / Q$.

In the case where $Q$ is a parabolic subgroup of $G$, a different proof of this result, based on ideas developed in [Ma], is given in [GM].

In the last decade or so, there have been intensive studies on the equidistribution properties of lattice points on homogeneous spaces of $G$ using various methods from analytic number theory, harmonic analysis, and ergodic theory (see [DRS], [EM], [EMM], [EMS], [GO], [EO], [Go], [L], [Ma], [No], etc.). Of particular interest is the case in which the homogeneous space is a real algebraic variety. While most of the attention in this direction is focused on the case of affine homogeneous varieties, not as much work has been done for the projective homogeneous varieties, except for the works [Go] and [Ma]. In [Go], the subject is the distribution of lattice orbits on the real projective homogeneous varieties of $G=\mathrm{SL}_{n}(\mathbb{R})$ with respect to the norm given by $\|g\|=\sqrt{\sum g_{i j}^{2}}, g \in \mathrm{SL}_{n}(\mathbb{R})$. In [Ma], Maucourant investigates the distribution of lattice orbits on the boundary of a real hyperbolic space. Corollary 1.7 extends both results by proving that an orbit of a lattice in a connected noncompact semisimple real algebraic group $G$ is equidistributed on any projective homogeneous variety of $G$ (with respect to a Riemannian metric).

More generally, we state the following conjecture.

\section{CONJECTURE 1.8}

Let $G$ be a connected semisimple Lie group with finite center, let $\Gamma$ be a lattice in $G$, and let $Y$ be a compact homogeneous space of $G$. Then every dense orbit of $\Gamma$ in $Y$ is equidistributed; that is, there exists a smooth measure $v$ on $Y$ such that for any $y \in Y$ with $\overline{\Gamma y}=Y$ and for any Borel set $\Omega \subset Y$ with boundary of measure zero,

$$
\#\{\gamma \in \Gamma: \gamma y \in \Omega, d(K, K \gamma)<T\} \sim_{T \rightarrow \infty} v(\Omega) \cdot \frac{\operatorname{Vol}\left(G_{T}\right)}{\operatorname{Vol}(G / \Gamma)} .
$$

The structure of compact homogeneous spaces of $G$ was studied in [Wit]. We note that the case of the conjecture when $Y=G / \Gamma$ for a cocompact lattice is also known (see Theorem 1.9).

\subsection{Measure-preserving lattice actions}

Let $G$ be a connected semisimple noncompact Lie group with finite center, and let $\Gamma_{1}, \Gamma_{2}$ be lattices in $G$. We consider the action of $\Gamma_{1}$ on $G / \Gamma_{2}$. Let $d$ be an invariant Riemannian metric on the symmetric space $K \backslash G$. 


\section{THEOREM 1.9}

Suppose that for $y \in G / \Gamma_{2}$, the orbit $\Gamma_{1} y$ is dense in $G / \Gamma_{2}$. Then for any $g \in G$ and any Borel subset $\Omega \subset G / \Gamma_{2}$ with boundary of measure zero,

$$
\left\{\gamma \in \Gamma_{1}: \gamma y \in \Omega, d(K, K g \gamma)<T\right\} \sim_{T \rightarrow \infty} \frac{\operatorname{Vol}(\Omega) \cdot \operatorname{Vol}\left(G_{T}\right)}{\operatorname{Vol}\left(G / \Gamma_{1}\right) \operatorname{Vol}\left(G / \Gamma_{2}\right)},
$$

where all volumes are computed with respect to one fixed Haar measure on G.

For example, Theorem 1.9 applies to the case where $G$ is a simple connected noncompact Lie group and where $\Gamma_{1}$ and $\Gamma_{2}$ are noncommensurable lattices in $G$. (Recall that the lattices are called commensurable if $\Gamma_{1} \cap \Gamma_{2}$ has finite index in both $\Gamma_{1}$ and $\Gamma_{2}$.) It was first observed by Vatsal [V] that $\Gamma_{1} \Gamma_{2}$ is dense in $G / \Gamma_{2}$. This is a (simple) consequence of Ratner's topological rigidity theorem [R2, Theorem 4].

Theorem 1.9 was proved in $[\mathrm{O}]$ for $G=\mathrm{SL}_{n}(\mathbb{R})$ equipped with the norm $\|g\|=$ $\sqrt{\sum g_{i j}^{2}}$ and was also proved in [GW] for general semisimple Lie groups without compact factors.

\section{Main ingredients of the proofs}

\subsection{The strong wavefront lemma}

The following theorem is a basic tool that enables us to reduce the counting problems for $\Gamma$ (as in Theorems 1.2,1.6) to the study of continuous flows on the homogeneous space $\Gamma \backslash G$.

Let $G$ be a connected noncompact semisimple Lie group with finite center, let $G=K A^{+} K$ be a Cartan decomposition, and let $M$ be the centralizer of $A$ in $K$.

THEOREM 2.1 (The strong wavefront lemma)

Let $\mathscr{C}$ be any closed subset of $A^{+}$with a positive distance from the walls of $A^{+}$. Then for any neighborhoods $U_{1}, U_{2}$ of $e$ in $K$ and $V$ of $e$ in $A$, there exists a neighborhood (1) of $e$ in $G$ such that for any $g=k_{1} a k_{2} \in K \mathscr{C} K$,

(1) $g \mathcal{O} \subset\left(k_{1} U_{1}\right)(a V M)\left(k_{2} U_{2}\right)$, and

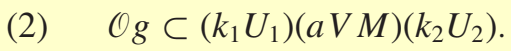

The strong wavefront lemma has been recently generalized to affine symmetric spaces in $[\mathrm{GOS}]$.

\section{Remark 2.2}

One can check that Theorem 2.1 fails if the set $\mathscr{C}$ contains a sequence that converges to a point in a wall of the Weyl chamber $A^{+}$. 
Theorem 2.1 has several geometric implications for the symmetric space $K \backslash G$, which are explained below.

(i) Strengthening of the wavefront lemma. Recall that the wavefront lemma introduced by Eskin and McMullen in [EM] says that for any neighborhood $\mathcal{O}^{\prime}$ of $e$ in $G$, there exists a neighborhood $\mathcal{O}$ of $e$ in $G$ such that

$$
a \mathcal{O} \subset \mathcal{O}^{\prime} a K \quad \text { for all } a \in A^{+} .
$$

To see that our strong wavefront lemma (1) implies the wavefront lemma for $a \in A^{+}$ with at least a fixed positive distance from the walls of $A^{+}$, note that $\mathcal{O}^{\prime}$ contains $U_{1} V$ for some neighborhood $U_{1}$ of $e$ in $K$ and some neighborhood $V$ of $e$ in $A$, and hence

$$
U_{1} a V M K=U_{1} V a K \subset \mathcal{O}^{\prime} a K .
$$

By Theorem 2.1(1), there exists a neighborhood $\mathcal{O}$ of $e$ such that

$$
a \mathcal{O} \subset U_{1} a V M K \text {. }
$$

Thus, $a \mathcal{O} \subset \mathcal{O}^{\prime} a K$.

To illustrate the geometric meaning of the strong wavefront lemma (1), we consider the unit disc $\mathbb{D}$ equipped with the standard hyperbolic metric and we consider the geodesic flow $g_{t}$ on the unit tangent bundle $\mathrm{T}^{1}(\mathbb{D})$ which transports a vector distance $t$ along the geodesic to which it is tangent. Note that with the identification $\mathrm{T}^{1}(\mathbb{D}) \simeq \mathrm{PSL}_{2}(\mathbb{R})$, the geodesic flow $g_{t}$ corresponds to the left multiplication by $\left(\begin{array}{cc}e^{t / 2} & 0 \\ 0 & e^{-t / 2}\end{array}\right)$. Let $p \in \mathbb{D}$, and let $K \subset \mathrm{T}^{1}(\mathbb{D})$ be the preimage of $p$ under the projection map $\pi: \mathrm{T}^{1}(\mathbb{D}) \rightarrow \mathbb{D}$. Note that $K$ consists of vectors lying over $p$ and pointing in all possible directions and that $g_{t}(K)$ consists of the unit vectors normal to the sphere $S_{t}(p) \subset \mathbb{D}$ of radius $t$. The wavefront lemma (see equation (2.3)) implies that one can find a neighborhood $\mathcal{O} \subset \mathrm{T}^{1}(\mathbb{D})$ of a vector $v$ based at $p$ such that $g_{t}(\mathcal{O})$ remains close to $g_{t}(K)$ uniformly for every $t \geq 0$.

However, this does not compare $g_{t}(\mathcal{O})$ with the vector $g_{t}(v)$ but rather with the set $g_{t}(K)$. Theorem 2.1(1) says that we may choose a neighborhood $\mathcal{O}$ of $v$ in $\mathrm{T}^{1}(\mathbb{D})$ so that $g_{t}(\mathcal{O})$ is close to the vector $g_{t}(v)$ uniformly on $t$ in both angular and radial components (see Figure 2).

(ii) Uniform openness of the map $K \times A^{+} \times K \rightarrow G$. The product map

$$
K \times\left(\text { interior of } A^{+}\right) \times K \rightarrow G
$$

is a diffeomorphism onto a dense open subset in $G$, and in particular, it is an open map. Theorem 2.1(2) shows that this map is uniformly open with respect to the base of neighborhoods $\mathcal{O}$, where $\mathcal{O}$ is a neighborhood of $e$ in $G$ and $g \in G$, on any subset contained in $K \times A^{+} \times K$ with a positive distance from the walls of $A^{+}$. 


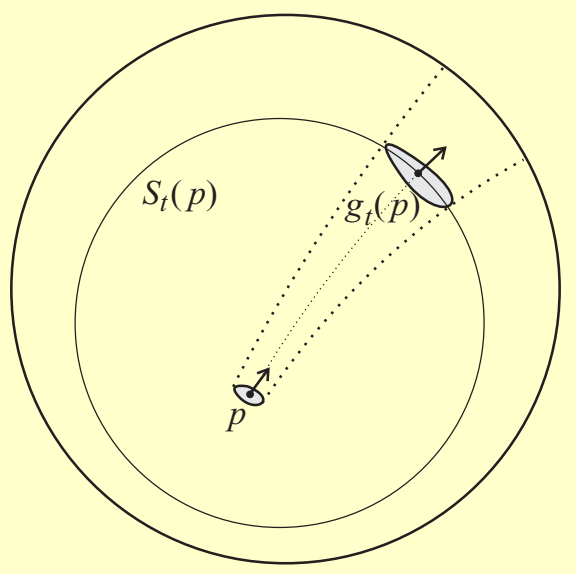

Figure 2

We illustrate the geometric meaning of this property for the case of the hyperbolic unit disc $\mathbb{D}$. It follows from Theorem 2.1(2) that for every neighborhood $\mathcal{O}^{\prime}$ of $e$ in $G$, there exists a neighborhood $\mathcal{O}$ of $e$ in $G$ such that for every $a \in A^{+}$with at least a fixed positive distance from the walls of $A^{+}$,

$$
\mathcal{O} a \subset K a \mathcal{O}^{\prime}
$$

This implies that for every open subset $\mathcal{O}^{\prime} \subset \mathrm{T}^{1}(\mathbb{D}), p \in \mathcal{O}^{\prime}$, and any $t>0$, the set $\pi\left(g_{t}\left(\mathcal{O}^{\prime}\right)\right)$ contains a ball centered at $\pi\left(g_{t}(p)\right)$ with a radius independent of $t$.

(iii) Well-roundness of bisectors. Theorem 2.1(2) also implies the following corollary, which is a generalization of the well-known property that the Riemannian balls are well rounded (a terminology used in [EM]). Note that the Riemannian ball $\{g \in$ $G: d(K, K g)<T\}$ is of the form $K A_{T}^{+} K$, where $A_{T}^{+}=\left\{a \in A^{+}: d(K, K a)<T\right\}$.

\section{COROLLARY 2.4}

For Borel subsets $\Omega_{1}, \Omega_{2} \subset K$ whose boundary has measure zero, the family $\left\{\Omega_{1} A_{T}^{+} \Omega_{2}: T>0\right\}$ of bisectors is well rounded; that is, for every $\varepsilon>0$, there exists a neighborhood 10 of $e$ in $G$ such that

$$
\operatorname{Vol}\left(\mathcal{O} \cdot \partial\left(\Omega_{1} A_{T}^{+} \Omega_{2}\right)\right) \leq \varepsilon \cdot \operatorname{Vol}\left(\Omega_{1} A_{T}^{+} \Omega_{2}\right)
$$

for all $T>0$.

\subsection{Uniform distribution of solvable flows}

Using the strong wavefront lemma, Theorem 1.2 is deduced from Theorem 2.5, which is also of interest from the viewpoint of ergodic theory. 
Let $K$ be a maximal compact subgroup of $G$ with the probability Haar measure v. Let $Q$ be a closed subgroup of $G$ containing a maximal connected split solvable subgroup of $G$, and let $\rho$ be a right-invariant Haar measure on $Q$. Fix a Cartan decomposition $G=K A^{+} K$ and $g \in G$. For $T>0$ and a subset $\Omega \subset K$, we define

$$
Q_{T}(g, \Omega)=\left\{q \in Q: q \in g^{-1} K A^{+} \Omega, d(K, K g q)<T\right\} .
$$

If $\Omega=K$ and $g=e$, the set $Q_{T}(g, \Omega)$ is simply $\{q \in Q: d(K, K q)<T\}$. Recall that $G_{n}$ denotes the product of all noncompact simple factors of $G$.

\section{THEOREM 2.5}

Let $G$ be realized as a closed subgroup of a Lie group L. Let $\Lambda$ be a lattice in $L$. Suppose that for $y \in \Lambda \backslash L$, the orbit $y G_{n}$ is dense in $\Lambda \backslash L$. Then for any Borel subset $\Omega \subset K$ with boundary of measure zero and $f \in C_{c}(\Lambda \backslash L)$,

$$
\lim _{T \rightarrow \infty} \frac{1}{\rho\left(Q_{T}(g, K)\right)} \int_{Q_{T}(g, \Omega)} f\left(y q^{-1}\right) d \rho(q)=\frac{\nu(M \Omega)}{\mu(\Lambda \backslash L)} \int_{\Lambda \backslash L} f d \mu,
$$

where $M$ is the centralizer of $A$ in $K$ and $\mu$ is an $L$-invariant measure on $\Lambda \backslash L$.

A main ingredient of the proof of Theorem 2.5 is the work of Shah [S] (see also Theorem 6.2) on the distribution in $\Lambda \backslash L$ of translates $y U g$ as $g \rightarrow \infty$ for a subset $U \subset K$. Shah's result is based on Ratner's classification of measures invariant under unipotent flows (see [R1]) and on the work of Dani and Margulis on the behavior of unipotent flows (see [DM]). Implementation of Shah's theorem in our setting is based on the fundamental property of the Furstenberg boundary $\mathscr{B}$ of $G$ : every regular element in a positive Weyl chamber acts on an open subset of full measure in $\mathscr{B}$ as a contraction.

\section{Remark 2.6 (On the rate of convergence)}

The method of [S, proof of Theorem 6.2] does not give any estimate on the rate of convergence. In the case where $L=G$ and $U=K$, Theorem 6.2 was proved by Eskin and McMullen [EM, Theorem 1.2]. The latter proof is based on the decay of the matrix coefficients of the quasi-regular representation of $G$ on $L^{2}(\Gamma \backslash G)$ and provides an estimate on the rate of convergence. Combining the strong wavefront lemma (Theorem 2.1) with the method from [EM], we can derive an estimate for the rate of convergence in Theorem 2.5 when $L=G$, provided that one knows the rate of decay of matrix coefficients of $L^{2}(\Gamma \backslash G)$. In this case, it is also possible to obtain rates of convergence for the theorems stated in the introduction. We hope to address this problem in a sequel to this article. 


\subsection{Equidistribution of lattice points in bisectors}

For $\Omega_{1}, \Omega_{2} \subset K$, and $g \in G$, we define

$$
G_{T}\left(g, \Omega_{1}, \Omega_{2}\right)=\left\{h \in G: h \in g^{-1} \Omega_{1} A^{+} \Omega_{2}, d(K, K g h)<T\right\} .
$$

Using the strong wavefront lemma (Theorem 2.1(2)), Theorem 1.6 is reduced to showing that the sets $G_{T}\left(g, \Omega_{1}, \Omega_{2}\right)$ are equidistributed in $\Gamma \backslash G$ in the sense of Theorem 2.5 for any Borel subsets $\Omega_{1}, \Omega_{2} \subset K$ with boundaries of measure zero.

\section{Cartan decomposition and the strong wavefront lemma}

Let $G$ be a connected noncompact semisimple Lie group with finite center, let $K$ be a maximal compact subgroup of $G$, and let $G=K \exp (\mathfrak{p})$ be the Cartan decomposition determined by $K$. A split Cartan subgroup $A$ with respect to $K$ is a maximal connected abelian subgroup of $G$ contained in $\exp (\mathfrak{p})$. It is well known that two split Cartan subgroups with respect to $K$ are conjugate to each other by an element of $K$. Fix a split Cartan subgroup $A$ of $G$ (with respect to $K$ ) with the set of positive roots $\Phi^{+}$ and the positive Weyl chamber

$$
A^{+}=\left\{a \in A: \alpha(\log a) \geq 0 \text { for all } \alpha \in \Phi^{+}\right\} .
$$

Set $\mathfrak{a}=\log (A)$, and set $\mathfrak{a}^{+}=\log \left(A^{+}\right)$. Let $M$ be the centralizer of $A$ in $K$. Note that $M$ is finite if and only if $G$ is real split.

The following lemma is well known (see, e.g., [K, Chapter V]).

LEMMA 3.1 (Cartan decomposition)

For every $g \in G$, there exists a unique element $\mu(g) \in \log \left(A^{+}\right)$such that $g \in$ $K \exp (\mu(g)) K$. Moreover, if $k_{1} a k_{2}=k_{1}^{\prime} a k_{2}^{\prime}$ for some $a$ in the interior of $A^{+}$, then there exists $m \in M$ such that $k_{1}=k_{1}^{\prime} m, k_{2}=m^{-1} k_{2}^{\prime}$.

Denote by $d$ an invariant Riemannian metric on the symmetric space $K \backslash G$.

\section{LEMMA 3.2}

For every $a_{1}$ and $a_{2}$ in the interior of $A^{+}$and $k \in K$,

$$
d\left(K a_{1}, K a_{2}\right) \leq d\left(K a_{1} k, K a_{2}\right) .
$$

\section{Proof}

Let $a_{i}=\exp \left(H_{i}\right)$ for $H_{i} \in \mathfrak{a}^{+}, i=1,2$. Then $K a_{1} k=K \exp \left(\operatorname{Ad}\left(k^{-1}\right) H_{1}\right)$. Applying the cosine inequality (see [H, Chapter I, Corollary 13.2]) to the geodesic triangle with 
vertices $K e, K a_{1} k$, and $K a_{2}$, we obtain

$$
\begin{aligned}
d\left(K a_{1} k, K a_{2}\right)^{2} & \geq d\left(K, K a_{1} k\right)^{2}+d\left(K, K a_{2}\right)^{2}-2 d\left(K, K a_{1} k\right) d\left(K, K a_{2}\right) \cos \alpha \\
& =\left\|H_{1}\right\|^{2}+\left\|H_{2}\right\|^{2}-2\left\|H_{1}\right\|\left\|H_{2}\right\| \cos \alpha,
\end{aligned}
$$

where $\alpha$ is the angle at the vertex $K e$. Since

$$
\cos \alpha=\frac{\left\langle\operatorname{Ad}\left(k^{-1}\right) H_{1}, H_{2}\right\rangle}{\left\|H_{1}\right\| \cdot\left\|H_{2}\right\|}
$$

and, by Lemma 3.3,

$$
\left\langle\operatorname{Ad}\left(k^{-1}\right) H_{1}, H_{2}\right\rangle \leq\left\langle H_{1}, H_{2}\right\rangle,
$$

it follows that

$$
d\left(K a_{1} k, K a_{2}\right)^{2} \geq\left\|H_{1}\right\|^{2}+\left\|H_{2}\right\|^{2}-2\left\langle H_{1}, H_{2}\right\rangle=\left\|H_{1}-H_{2}\right\|^{2}=d\left(K a_{1}, K a_{2}\right)^{2} .
$$

The lemma is proved.

LEMMA 3.3

For any $H_{1}$ and $H_{2}$ in the interior of $\mathfrak{a}^{+}$and for any $k \in K$,

$$
\left\langle H_{1}, H_{2}\right\rangle \geq\left\langle\operatorname{Ad}(k) H_{1}, H_{2}\right\rangle \text {. }
$$

\section{Proof}

By [H, Chapter VIII, Proposition 5.2] and its proof, every $G$-invariant positive definite form on $K \backslash G$ is of the form $\sum_{i} \alpha_{i} B_{i}$, where $B_{i}$ 's are the Killing forms of the simple factors of $G$ and $\alpha_{i}>0$. Thus, it is sufficient to consider the case when $G$ is simple, and the Riemannian metric is given by the Killing form $B$ of $G$.

Define the function $f(k)=\left\langle\operatorname{Ad}(k) H_{1}, H_{2}\right\rangle$ on $K$. Let $k_{0} \in K$ be a point where $f$ attains its maximum. For every $Z \in \operatorname{Lie}(K)$,

$$
\begin{aligned}
0 & =\left.\frac{d}{d t}\right|_{t=0} f\left(k_{0} e^{t Z}\right)=\left.\frac{d}{d t}\right|_{t=0} B\left(\operatorname{Ad}\left(k_{0}\right) \operatorname{Ad}\left(e^{t Z}\right) H_{1}, H_{2}\right) \\
& =B\left(\left.\operatorname{Ad}\left(k_{0}\right) \frac{d}{d t}\right|_{t=0} \operatorname{Ad}\left(e^{t Z}\right) H_{1}, H_{2}\right)=B\left(\operatorname{Ad}\left(k_{0}\right)\left(\operatorname{ad}(Z) H_{1}\right), H_{2}\right) \\
& =B\left(\operatorname{Ad}\left(k_{0}\right)\left[Z, H_{1}\right], H_{2}\right)=B\left(\operatorname{Ad}\left(k_{0}\right) Z,\left[\operatorname{Ad}\left(k_{0}\right) H_{1}, H_{2}\right]\right) .
\end{aligned}
$$

This shows that $\left[\operatorname{Ad}\left(k_{0}\right) H_{1}, H_{2}\right] \perp \operatorname{Lie}(K)$. Since $\left[\operatorname{Ad}\left(k_{0}\right) H_{1}, H_{2}\right] \in \operatorname{Lie}(K)$ and the restriction of $B$ to $\operatorname{Lie}(K)$ is negative definite, it follows that $\left[\operatorname{Ad}\left(k_{0}\right) H_{1}, H_{2}\right]=0$. Therefore, $\operatorname{Ad}\left(k_{0}\right) H_{1} \in \mathfrak{a}$. Since the Weyl group $W$ acts transitively on the set of 
Weyl chambers in $A$, and since $K$ contains all representatives of the Weyl group, there exists an element $w \in K$ which normalizes $A$ such that $\operatorname{Ad}\left(w^{-1} k_{0}\right) H_{1} \in \mathfrak{a}^{+}$. Since $H_{1}$ is in the interior of $\mathfrak{a}^{+}$, it follows from the uniqueness of the Cartan decomposition (Lemma 3.1) that $\operatorname{Ad}\left(k_{0}\right) H_{1}=\operatorname{Ad}(w) H_{1}$. It is easy to see from [H, page 288] that $\left\|\operatorname{Ad}(w) H_{1}-H_{2}\right\|, w \in W$, achieves its minimum at $w=e$. This implies that $\left\langle\operatorname{Ad}(w) H_{1}, H_{2}\right\rangle$ is maximal for $w=e$ and finishes the proof.

PROPOSITION 3.4

Let $\mathscr{C}$ be a closed subset contained in $A^{+}$with a positive distance from the walls of $A^{+}$. Then for any neighborhood $U_{0}$ of $e$ in $K$, there exists $\varepsilon>0$ such that for any $a \in \mathscr{C}$,

$$
\{k \in K: d(K a k, K a)<\varepsilon\} \subset M U_{0} .
$$

\section{Proof}

Denote by $\Pi$ the set of simple roots corresponding to the Weyl chamber $A^{+}$. Without loss of generality, we may assume that

$$
\mathscr{C}=\left\{a \in A^{+}: \alpha(\log a) \geq C \text { for all } \alpha \in \Pi\right\}
$$

for some $C>0$. Suppose that, instead, there exist sequences $\left\{a_{i}\right\} \subset \mathscr{C}$ and $\left\{k_{i}\right\} \subset K$ such that $d\left(K a_{i} k_{i}, K a_{i}\right) \rightarrow 0$ as $i \rightarrow \infty$ and that no limit points of $\left\{k_{i}\right\}$ are contained in $M$. Passing to a subsequence, we may assume that $k_{i} \rightarrow k_{0}$ as $i \rightarrow \infty$ for some $k_{0} \in K-M$ and that for every $\alpha \in \Phi$, the sequence $\left\{\alpha\left(\log a_{i}\right)\right\}$ is either bounded or divergent. Set

$$
\begin{aligned}
& \Phi_{ \pm}=\left\{\alpha \in \Phi: \alpha\left(\log a_{i}\right) \rightarrow \pm \infty \text { as } i \rightarrow \infty\right\}, \\
& \Phi_{0}=\left\{\alpha \in \Phi:\left\{\alpha\left(\log a_{i}\right)\right\} \text { is bounded }\right\} .
\end{aligned}
$$

Let $P^{+}$be the standard parabolic subgroup associated to $\Pi-\Phi_{+}$, and let $P^{-}$be the standard opposite parabolic subgroup for $P^{+}$. Note that

$$
P^{-}=\left\{g \in G:\left\{a_{i} g a_{i}^{-1}\right\} \text { is bounded }\right\} .
$$

Denote by $U^{+}$and $U^{-}$the unipotent radicals of $P^{+}$and $P^{-}$, respectively. Set $Z=$ $P^{+} \cap P^{-}$, so that $P^{ \pm}=Z U^{ \pm}$. It is easy to see that $P^{ \pm} \cap K \subset Z$. Denote by $\mathfrak{g}, \mathfrak{u}^{+}$, $\mathfrak{u}^{-}, \mathfrak{z}$ the corresponding Lie algebras, and denote by $\mathfrak{g}_{\alpha}, \alpha \in \Phi$ the root subspaces in g. We have

$$
\mathfrak{u}^{ \pm}=\bigoplus_{\alpha \in \Phi_{ \pm}} \mathfrak{g}_{\alpha} \quad \text { and } \quad \mathfrak{z}=\bigoplus_{\alpha \in \Phi_{0}} \mathfrak{g}_{\alpha}
$$


Step 1. We claim that $k_{0} \in Z$.

There is an embedding $\pi: G / C_{G} \rightarrow \operatorname{SL}_{d}(\mathbb{R})$, where $C_{G}$ is the center of $G$, such that $\pi\left(A C_{G}\right)$ is contained in the group of diagonal matrices. We have

$$
\pi\left(a_{i} k_{i} a_{i}^{-1}\right)_{s t}=\pi\left(a_{i}\right)_{s s} \pi\left(a_{i}\right)_{t t}^{-1} \cdot \pi\left(k_{i}\right)_{s t}, \quad s, t=1, \ldots, d .
$$

Passing to a subsequence, we may assume that for each $(s, t)$, the sequence $\left\{\pi\left(a_{i}\right)_{s s} \pi\left(a_{i}\right)_{t t}^{-1}\right\}$ is either bounded or divergent. If the sequence $\left\{\pi\left(a_{i}\right)_{s s} \pi\left(a_{i}\right)_{t t}^{-1}\right\}$ is divergent, then $\pi\left(k_{i}\right)_{s t} \rightarrow 0$ as $i \rightarrow \infty$. Thus, $\pi\left(k_{0}\right)_{s t}=0$ for every pair $(s, t)$ such that $\left\{\pi\left(a_{i}\right)_{s s} \pi\left(a_{i}\right)_{t t}^{-1}\right\}$ is divergent. It follows that $\pi\left(a_{i} k_{0} a_{i}^{-1}\right)$ is bounded. Since the center of $G$ is finite, this proves that the sequence $\left\{a_{i} k_{0} a_{i}^{-1}\right\}$ is bounded. Thus, $k_{0} \in P^{-}$. Since $P^{-} \cap K \subset Z$, the claim follows.

Step 2. We claim that $d\left(K a_{i} k_{0} a_{i}^{-1}, K\right) \rightarrow 0$ as $i \rightarrow \infty$.

Write $k_{i}=k_{0} l_{i}$, where $l_{i} \in K$ and $l_{i} \rightarrow e$ as $i \rightarrow \infty$. Then

$$
d\left(K a_{i} k_{i}, K a_{i}\right)=d\left(K a_{i} k_{0} a_{i}^{-1}, K a_{i} l_{i}^{-1} a_{i}^{-1}\right) \rightarrow 0 \quad \text { as } i \rightarrow \infty .
$$

Thus, the sequence $\left\{a_{i} l_{i}^{-1} a_{i}^{-1}\right\}$ is bounded, and hence, we may assume that it converges. Since $l_{i}^{-1} \rightarrow e$ as $i \rightarrow \infty$ and $\mathfrak{g}=\mathfrak{u}^{-} \oplus \mathfrak{z} \oplus \mathfrak{u}^{+}$, we obtain that $l_{i}^{-1}=u_{i}^{-} z_{i} u_{i}^{+}$for some $u_{i}^{-} \in U^{-}, z_{i} \in Z$, and $u_{i}^{+} \in U^{+}$such that $u_{i}^{-} \rightarrow e, z_{i} \rightarrow e$, and $u_{i}^{+} \rightarrow e$ as $i \rightarrow \infty$. It follows from (3.5) that $a_{i} u_{i}^{-} a_{i}^{-1} \rightarrow e$ and $a_{i} z_{i} a_{i}^{-1} \rightarrow e$ as $i \rightarrow \infty$. Hence, $a_{i} l_{i}^{-1} a_{i}^{-1} \rightarrow u^{+}$as $i \rightarrow \infty$ for some $u^{+} \in U^{+}$. On the other hand, by Step 1 , passing to a subsequence, we get $a_{i} k_{0} a_{i}^{-1} \rightarrow z$ as $i \rightarrow \infty$ for some $z \in Z$. Thus, by (3.6), $z^{-1} u^{+} \in K$ as $i \rightarrow \infty$. Since $P^{+} \cap K \subset Z$, we deduce that $u^{+}=e$. Hence, $a_{i} l_{i}^{-1} a_{i} \rightarrow e$ as $i \rightarrow \infty$, and the claim follows from (3.6).

Step 3. We claim that $a_{i} k_{0} a_{i}^{-1}=d_{i} k_{0} d_{i}^{-1}$ for some bounded sequence $\left\{d_{i}\right\} \subset \mathscr{C}$.

Recall that the system of simple roots $\Pi$ is a basis of the dual space of the Lie algebra of $A$. Hence, we may write $a_{i}=b_{i} c_{i}$, where $b_{i}, c_{i} \in A^{+}$such that $\alpha\left(\log b_{i}\right)=0$ for every $\alpha \in \Pi \cap \Phi_{+}$and $\alpha\left(\log c_{i}\right)=0$ for every $\alpha \in \Pi-\Phi_{+}$. Then $c_{i}$ commutes with $Z$, and since $\alpha\left(b_{i}\right)=\alpha\left(a_{i}\right)$ for every $\alpha \in \Pi-\Phi_{+}$, the sequence $\left\{b_{i}\right\}$ is bounded. Let $c_{0} \in A^{+}$be such that $\alpha\left(\log c_{0}\right)=C$ for every $\alpha \in \Pi \cap \Phi_{+}$and $\alpha\left(\log c_{0}\right)=0$ for every $\alpha \in \Pi-\Phi_{+}$. Then $d_{i}=b_{i} c_{0} \in \mathscr{C}$ and $a_{i} k_{0} a_{i}^{-1}=d_{i} k_{0} d_{i}^{-1}$, as required.

Taking a subsequence, we obtain that $d_{i} \rightarrow d_{0}$ as $i \rightarrow \infty$ for some $d_{0} \in \mathscr{C}$ and $d_{0} k_{0} d_{0}^{-1} \in K$. This implies that $d_{0} k_{0}=k d_{0}$ for some $k \in K$. Since $d_{0}$ lies in the interior of $A^{+}$, it follows from the uniqueness properties of the Cartan decomposition (Lemma 3.1) that $k_{0}=k \in M$. This is a contradiction. 
THEOREM 3.7 (The strong wavefront lemma)

Let $\mathscr{C}$ be a closed subset contained in $A^{+}$with a positive distance from the walls of $A^{+}$. Then for any neighborhoods $U$ of $e$ in $K$ and $V$ of e in $A$, there exists a neighborhood (1) of $e$ in $G$ such that

$$
\mathcal{O} \cup g \mathcal{O} \subset\left(k_{1} U\right)(a V M)\left(k_{2} U\right)
$$

for any $g=k_{1} a k_{2} \in K \mathscr{C} K$.

Proof

Without loss of generality, we may assume that $\mathscr{C}=A^{+}(C)$ for some $C>0$, where

$$
A^{+}(C)=\left\{a \in A^{+}: \alpha(\log a) \geq C \text { for all } \alpha \in \Phi^{+}\right\} .
$$

Replacing $V$ by a smaller subset if necessary, we may assume that $A^{+}(C) V$ is contained in the interior of $A^{+}(C / 2)$.

Step 1. We claim that there exists an $\varepsilon$-neighborhood $\mathcal{O}$ of $e$ in $G$ such that

$$
\mathcal{O} g \subset K a V K \quad \text { for all } g=k_{1} a k_{2} \in K A^{+}(C) K \text {. }
$$

We take $\varepsilon>0$ to be sufficiently small so that the $\varepsilon$-neighborhood of $e$ in $A$ is contained in $V$. Let $\mathcal{O}$ be the $\varepsilon$-neighborhood of $e$ in $G$. For $h \in \mathcal{O} a$, write $h=k_{1}^{\prime} b k_{2}^{\prime} \in K A^{+} K$. Then $d\left(K b k_{2}^{\prime} a^{-1}, K\right)=d\left(K b k_{2}^{\prime}, K a\right)<\varepsilon$. Since $d(K b, K a) \leq d\left(K b k_{2}^{\prime}, K a\right)$, by Lemma 3.2 we have

$$
d(K b, K a)<\varepsilon,
$$

and hence, $h=k_{1}^{\prime} b k_{2}^{\prime} \in K V a K$. This shows that $\mathcal{O} a \subset K a V K$. Since $\mathcal{O}$ is $K$ invariant,

$$
\mathcal{O} k_{1} a k_{2} \subset \mathcal{O} a k_{2} \subset K a V K
$$

This proves the claim.

Step 2. We claim that there exists an $\varepsilon$-neighborhood $\mathcal{O}$ of $e$ in $G$ such that

$$
\mathcal{O} g \subset K A^{+}\left(k_{2} U\right) \text { for all } g=k_{1} a k_{2} \in K A^{+}(C) K \text {. }
$$

Let $U_{0}$ be a neighborhood of $e$ in $K$ such that $k U_{0} k^{-1} \subset U$ for all $k \in K$. Choose $\varepsilon>0$, which satisfies Step 1 above, and Proposition 3.4 holds with respect to $2 \varepsilon, U_{0}$, and $A^{+}(C / 2)$. Take $\mathcal{O}$ to be the $\varepsilon$-neighborhood of $e$ in $G$. Then for any 
$h=k_{1}^{\prime} b k_{2}^{\prime} \in \mathcal{O} a$, we have, by (3.8),

$$
d\left(K b k_{2}^{\prime}, K b\right) \leq d\left(K b k_{2}^{\prime}, K a\right)+d(K b, K a)<2 \varepsilon
$$

Since $\mathcal{O} a \subset K A^{+}(C / 2) K$, by Proposition 3.4, this implies that $k_{2}^{\prime} \in M U_{0}$, and hence, O $a \subset K A^{+} M U_{0}$. Thus,

$$
\mathcal{O}\left(k_{1} a k_{2}\right) \subset K A^{+} M U_{0} k_{2} \subset K A^{+} M\left(k_{2} U\right) .
$$

Step 3. We claim that there exists a neighborhood $\mathcal{O}$ of $e$ in $G$ such that

$$
\mathcal{O} g \subset\left(k_{1} U\right) A^{+} K \quad \text { for all } g=k_{1} a k_{2} \in K A^{+}(C) K
$$

Recall that by the Iwasawa decomposition, $G=K A N$, where $N$ is the subgroup of $G$ corresponding to the sum of the positive-root spaces. Since the Weyl group acts transitively on the sets of simple roots, there exists an element $w \in K$ which normalizes $A$ such that $w A^{+}(C) w^{-1}=\left(A^{+}(C)\right)^{-1}$.

Let $U_{0}$ be a neighborhood of $e$ in $K$ so that $U_{0}\left(w U_{0}^{-1} w^{-1}\right) \subset U$. Let $\mathcal{O}$ be the neighborhood from Step 2 with respect to $U_{0}$, and let $\mathcal{O}_{1}$ be a neighborhood of $e$ in $A N$ so that $c^{-1} \mathcal{O}_{1} c \subset \mathcal{O}$ for all $c \in A^{+}$. Since $w \in K, w \mathcal{O} w^{-1}=\mathcal{O}$. Conjugating (3.9) (with respect to $U_{0}$ ) by $w$, we have, for any $b \in w A^{+}(C) w^{-1}$,

$$
\mathcal{O} b \subset K\left(A^{+}\right)^{-1}\left(w U_{0} w^{-1}\right) .
$$

For $a \in A^{+}(C), a^{-1} \in w A^{+}(C) w^{-1}$, and hence,

$$
a^{-1} \mathcal{O}_{1} \subset \mathcal{O} a^{-1} \subset K\left(A^{+}\right)^{-1}\left(w U_{0} w^{-1}\right) \text {. }
$$

By taking the inverse of (3.10),

$$
\mathcal{O}_{1}^{-1} a \subset\left(w U_{0}^{-1} w^{-1}\right) A^{+} K
$$

Since the product map $K \times A N \rightarrow G$ is a diffeomorphism, $U_{0} O_{1}^{-1}$ is a neighborhood of $e$ in $G$. Therefore, there exists a neighborhood $\mathcal{O}_{2}$ of $e$ in $G$ such that $k^{-1} \mathcal{O}_{2} k \subset U_{0} \mathcal{O}_{1}^{-1}$ for all $k \in K$. Then, by (3.11),

$$
\mathcal{O}_{2} k_{1} a k_{2} \subset k_{1} U_{0} \mathcal{O}_{1}^{-1} a k_{2} \subset k_{1} U_{0}\left(w U_{0}^{-1} w^{-1}\right) A^{+} K \subset k_{1} U A^{+} K
$$

This finishes the proof of Step 3.

By the above three steps, we obtain a neighborhood $\mathcal{O}_{1}$ of $e$ in $G$ such that for all $g=k_{1} a k_{2} \in K A^{+}(C) K$,

$$
\mathcal{O}_{1} g \subset K a V K \cap K A^{+}\left(k_{2} U\right) \cap\left(k_{1} U\right) A^{+} K
$$


By the uniqueness of the Cartan decomposition (Lemma 3.1),

$$
K a V K \cap K A^{+}\left(k_{2} U\right) \cap\left(k_{1} U\right) A^{+} K=\left(k_{1} U\right)(a V M)\left(k_{2} U\right) .
$$

Hence, we have shown that for every neighborhood $U$ of $e$ in $K$ and for every neighborhood $V$ of $e$ in $A$, there exists a neighborhood $\mathcal{O}_{1}$ of $e$ in $G$ such that

$$
\mathcal{O}_{1} g \subset\left(k_{1} U\right)(a V M)\left(k_{2} U\right) \text { for all } g=k_{1} a k_{2} \in K A^{+}(C) K .
$$

Let $U_{0}$ be a neighborhood of $e$ in $K$ such that $k U_{0}^{-1} k^{-1} \subset U$ for every $k \in K$, and let $V_{0}$ be a neighborhood of $e$ in $A$ such that $w V_{0}^{-1} w^{-1} \subset V$, where $w$ is the element of $K$ defined in Step 3. Let $\mathcal{O}_{2}$ be a neighborhood of $e$ in $G$ such that

$$
\mathcal{O}_{2} g \subset\left(k_{1} U_{0}\right)\left(a V_{0} M\right)\left(k_{2} U_{0}\right) \quad \text { for all } g=k_{1} a k_{2} \in K A^{+}(C) K .
$$

By taking the inverse, we have

$$
g \mathcal{O}_{2}^{-1} \subset\left(U_{0}^{-1} k_{1}\right)\left(a V_{0}^{-1} M\right)\left(U_{0}^{-1} k_{2}\right) \quad \text { for all } g=k_{1} a k_{2} \in K A^{+}(C)^{-1} K .
$$

Conjugating by $w$, we get

$$
g\left(w \mathcal{O}_{2}^{-1} w^{-1}\right) \subset\left(k_{1} U\right)(a V M)\left(k_{2} U\right) \quad \text { for all } g=k_{1} a k_{2} \in K A^{+}(C) K .
$$

Set $\mathcal{O}=\mathcal{O}_{1} \cap\left(w \mathcal{O}_{2}^{-1} w^{-1}\right)$ to finish the proof.

\section{Contractions on $G / B$}

Let $G$ be a connected noncompact semisimple Lie group with finite center, let $K$ be a maximal compact subgroup of $G$, and let $B$ be a maximal connected split solvable subgroup of $G$. Let $N$ be the unipotent radical of $B$. The normalizer $P$ of $N$ in $G$ is the unique minimal parabolic subgroup of $G$ containing $B$.

\section{LEMMA 4.1}

There exist a split Cartan subgroup $A$ of $G$ with respect to $K$ and an ordering on the root system $\Phi$ such that

- $G=K A^{+} K$,

- $B=A N$,

- $\quad N$ is the subgroup generated by all positive-root subgroups of $G$ with respect to $A$, and

- $\quad P=M A N$, where $M$ is the centralizer of $A$ in $K$ and $M=K \cap P$.

\section{Proof}

Take any split Cartan subgroup $A_{0}$ and a Weyl chamber $A_{0}^{+}$so that the Cartan decomposition $G=K A_{0}^{+} K$ holds. Set $P_{0}=M_{0} A_{0} N_{0}$, where $M_{0}$ is the centralizer of $A_{0}$ in 
$K$ and $N_{0}$ is the subgroup generated by all positive-root subgroups of $G$ with respect to $A_{0}$. Note that $P_{0}$ is a minimal parabolic subgroup of $G$ (see [W, Section 1.2.3]). By the Iwasawa decomposition, $G=K P_{0}$. Since all minimal parabolic subgroups are conjugate to each other, there exists $k \in K$ such that $P=k P_{0} k^{-1}$. Set $A=k A_{0} k^{-1}$, $A^{+}=k A_{0}^{+} k^{-1}$, and $M=k M_{0} k^{-1}$. It is clear that $N=k N_{0} k^{-1}$. Since $A N$ is normal in $P$, it is the unique maximal connected split solvable subgroup in $P$. Thus, $B=A N$.

Let $A$ be a split Cartan subgroup as in Lemma 4.1. Denote by $M$ the centralizer of $A$ in $K$, and denote by $N^{-}$the subgroup generated by all negative-root subgroups of $G$. One can check that the map

$$
N^{-} \times M \rightarrow G / B:(n, m) \mapsto n m B
$$

is a diffeomorphism onto its image. Moreover, it follows from the properties of Bruhat decomposition that $G / B-\pi\left(N^{-} \times M\right)$ is a finite union of closed submanifolds of smaller dimensions. On the other hand, by the Iwasawa decomposition, the map

$$
K \rightarrow G / B: k \mapsto k B
$$

is a diffeomorphism. Thus, we have a map $N^{-} \times M \rightarrow K$. Since $M$ normalizes $B$, this map is right- $M$-equivariant. Denote by $S \subset K$ the submanifold that is the image of $N^{-} \times\{e\}$ under this map.

Let $v$ be the probability Haar measure on $K$, and let $\tau$ be the probability Haar measure on $M$. Then $v=\sigma \otimes \tau$ for some finite smooth measure $\sigma$ on $S$ (see [W, page 73]); that is,

$$
\int_{K} f d v=\int_{M} \int_{S} f(s m) d \sigma(s) d \tau(m), \quad f \in C(K) .
$$

For $C>0$, put

$$
A^{+}(C)=\left\{a \in A: \alpha(\log a) \geq C \text { for all } \alpha \in \Phi^{+}\right\} .
$$

LEMMA 4.3

Let $\Omega$ be a Borel subset of $K, s \in S, m \in M$, and $k \in K$. For $a \in A, r \in K$, and $U \subset K$, define

$$
\Omega_{r}(k, a, U)=\left\{l \in \Omega: l k B \in a^{-1} r^{-1} U B\right\} .
$$


(1) Let $V W \subset K$ be an open neighborhood of $s$, where $V \subset S$ and $W \subset M$ are open subsets. Then for every $\varepsilon>0$, there exists $C>0$ such that

$$
v\left(\left(\Omega \cap m^{-1} S W k^{-1}\right)-\Omega_{s m}(k, a, V W)\right)<\varepsilon \quad \text { for all } a \in A^{+}(C) .
$$

(2) Let $U \subset K$ be a Borel subset such that $s \notin \overline{U M}$. Then for every $\varepsilon>0$, there exists $C>0$ such that

$$
\nu\left(\Omega_{s m}(k, a, U)\right)<\varepsilon \quad \text { for all } a \in A^{+}(C)
$$

\section{Proof}

Note that $\Omega_{s m}(k, a, U)=m^{-1} \cdot(m \Omega)_{s}(k, a, U)$. Hence, replacing $\Omega$ by $m \Omega$, we may assume that $m=e$. Also, $\Omega_{s}(k, a, U)=(\Omega k)_{s}(e, a, U) \cdot k^{-1}$. Thus, we may assume that $k=e$.

The proof is based on the observation that elements in the interior of $A^{+}$act on $N^{-} B$ as contractions (see [Z, Proposition 8.2.5]). Denote by $\pi$ the map $\pi: N^{-} \rightarrow$ $G / B: n \mapsto n B$. Note that for $a \in A$ and $V_{0} \subset S$,

$$
\pi^{-1}\left(a^{-1} V_{0} B\right)=a^{-1} \pi^{-1}\left(V_{0} B\right) a
$$

Let $D$ be a compact set in $S$ so that $\sigma(S-D)<\varepsilon / 2$.

Suppose that $s \in V W$. Let $W_{0} \subset W$ be a compact set so that $\tau\left(W-W_{0}\right)<$ $\varepsilon / 2$. Then $s^{-1} V W$ is a neighborhood of $W_{0}$. By uniform continuity, there exists a neighborhood $V_{0}$ of $e$ in $S$ such that $V_{0} W_{0} \subset s^{-1} V W$. For each $a \in A^{+}$, the map $N^{-} \rightarrow N^{-}: x \rightarrow a^{-1} x a$ expands any neighborhood of $e$ at least by the factor of $\min _{\alpha \in \Phi^{+}} e^{\alpha(\log a)}$. Hence, there exists $C>0$ such that $\pi^{-1}(D B) \subset a^{-1} \pi^{-1}\left(V_{0} B\right) a$ for all $a \in A^{+}(C)$. Then by (4.4), $D B \subset a^{-1} V_{0} B$. It follows that $D W_{0} B \subset a^{-1} V_{0} W_{0} B$ and that

$$
\begin{aligned}
v\left(\Omega_{s}(e, a, V W)\right) & \geq v\left(\Omega \cap D W_{0}\right) \geq v(\Omega \cap S W)-v((S-D) M)-v\left(S\left(W-W_{0}\right)\right) \\
& \geq v(\Omega \cap S W)-\varepsilon .
\end{aligned}
$$

This proves the first part of the lemma.

To prove the second part, we observe that there exists an open subset $V \subset S$ such that $s \in V \subset K-\overline{U M}$. Since $\nu\left(m^{-1} S M k^{-1}\right)=1$, it suffices to apply the first part with $W=M$.

\section{LEMMA 4.5}

Let $W \subset M$ and $\Omega \subset K$ be Borel subsets. Then for every $k \in K$, we have

$$
\int_{M} v\left(\Omega \cap m^{-1} S W k^{-1}\right) d \tau(m)=v(\Omega) \tau(W) .
$$




\section{Proof}

Without loss of generality, $k=e$. Since $M$ normalizes $N^{-}$, we have $m^{-1} S m \subset S$. Since $v(K-S M)=0$, we may assume that $\Omega \subset S M$. Moreover, it suffices to prove the lemma for $\Omega=\Omega_{S} \Omega_{M}$ with Borel sets $\Omega_{S} \subset S$ and $\Omega_{M} \subset M$. By (4.2),

$$
v\left(\Omega \cap m^{-1} S W\right)=\sigma\left(\Omega_{S}\right) \tau\left(\Omega_{M} \cap m^{-1} W\right) .
$$

For $Y \subset M$, denote by $\chi_{Y}$ the characteristic function of $Y$. We have

$$
\begin{gathered}
\int_{M} \tau\left(\Omega_{M} \cap m^{-1} W\right) d \tau(m)=\int_{M} \int_{M} \chi_{\Omega_{M}}(l) \chi_{m^{-1} W}(l) d \tau(l) d \tau(m) \\
=\int_{M} \chi_{\Omega_{M}}(l)\left(\int_{M} \chi_{W l^{-1}}(m) d \tau(m)\right) d \tau(l)=\tau\left(\Omega_{M}\right) \tau(W) .
\end{gathered}
$$

Since $v(\Omega)=\sigma\left(\Omega_{S}\right) \tau\left(\Omega_{M}\right)$, this proves the lemma.

\section{Volume estimates}

Let $G$ be a connected noncompact semisimple Lie group with finite center, and let $G=K A^{+} K$ be a Cartan decomposition. Let $d k$ denote the probability Haar measure on $K$, let $d t$ denote the Lebesgue measure on the Lie algebra $\mathfrak{a}$ of $A$, and let $d a$ denote the Haar measure on $A$ derived from $d t$ via the exponential map. We denote by $m$ the Haar measure on $G$ which is normalized so that for any $f \in C_{c}(G)$,

$$
\int_{G} f d m=\int_{K} \int_{A^{+}} \int_{K} f\left(k_{1} a k_{2}\right) \xi(\log a) d k_{1} d a d k_{2},
$$

where

$$
\xi(t)=\prod_{\alpha \in \Phi^{+}} \sinh (\alpha(t))^{m_{\alpha}}, \quad t \in \mathfrak{a},
$$

and $m_{\alpha}$ is the dimension of the root subspace corresponding to $\alpha$. In particular, for any measurable subset $D \subset A^{+}$, we have

$$
\operatorname{Vol}(K D K)=\int_{D} \xi(\log a) d a .
$$

Let $\|\cdot\|$ be a Euclidean norm on $\mathfrak{a}$; that is, for some basis $v_{1}, \ldots, v_{n}$ of $\mathfrak{a}$, $\left\|\sum_{i} c_{i} v_{i}\right\|=\sqrt{\sum_{i} c_{i}^{2}}$. We assume that $\|\cdot\|$ is invariant under the Weyl group action. For instance, $\|\cdot\|$ can be taken, to be the norm induced from an invariant Riemannian metric $d$ on the symmetric space $K \backslash G$; that is,

$$
\|t\|=d(K, K \exp (t)) \quad \text { for } t \in \mathfrak{a} .
$$


For $T>0$ and $\mathfrak{t} \subset \mathfrak{a}$, set

$$
\mathfrak{t}_{T}=\{t \in \mathfrak{t}:\|t\|<T\} .
$$

Let $\rho=(1 / 2) \sum_{\alpha \in \Phi^{+}} m_{\alpha} \alpha$. One can check that the maximum of $\rho$ on $\overline{\mathfrak{a}}_{1}$ is achieved at a unique point contained in the interior of $\mathfrak{a}^{+}$, which we call the barycenter of $\mathfrak{a}^{+}$.

We present a simple derivation of the asymptotics of the volume of Riemannian balls in a symmetric space of noncompact type (see also [Kn, Theorem 6.2]).

\section{LEMMA 5.4}

Let $\mathfrak{q}$ be a convex cone in $\mathfrak{a}^{+}$centered at the origin that contains the barycenter of $\mathfrak{a}^{+}$ in its interior. Then for some $C>0$ (independent of $\mathfrak{q}$ ),

$$
\int_{\mathfrak{q}_{T}} \xi(t) d t \sim_{T \rightarrow \infty} C \cdot T^{(r-1) / 2} e^{\delta T},
$$

where $r=\mathbb{R}-\operatorname{rank}(G)=\operatorname{dim} A$ and $\delta=\max \left\{2 \rho(t): t \in \overline{\mathfrak{a}}_{1}\right\}$. In particular,

$$
\int_{\mathfrak{q}_{T}} \xi(t) d t \sim_{T \rightarrow \infty} \int_{\mathfrak{a}_{T}^{+}} \xi(t) d t
$$

Proof

We have

$$
\int_{\mathfrak{q}_{T}} \xi(t) d t=\frac{1}{2^{\left|\Phi^{+}\right|}} \int_{\mathfrak{q}_{T}} e^{2 \rho(t)} d t+\text { other terms, }
$$

where the "other terms" are linear combinations of integrals of the form $\int_{\mathfrak{q}_{T}} e^{\lambda(t)} d t$ such that $2 \rho-\lambda=\sum_{\alpha \in \Phi^{+}} n_{\alpha} \alpha$ for some $n_{\alpha} \geq 0$. In particular, $2 \rho>\lambda$ in the interior of the Weyl chamber $\mathfrak{a}^{+}$. Since the maximum of $2 \rho$ in $\overline{\mathfrak{a}}_{1}$ is achieved in the interior of $\mathfrak{q}$, then

$$
\max \left\{2 \rho(t): t \in \overline{\mathfrak{q}}_{1}\right\}>\max \left\{\lambda(t): t \in \overline{\mathfrak{q}}_{1}\right\} \stackrel{\text { def }}{=} \delta^{\prime} .
$$

Thus,

$$
\int_{\mathfrak{q}_{T}} e^{\lambda(t)} d t \leq e^{\delta^{\prime} T} \operatorname{Vol}\left(\mathfrak{q}_{T}\right) \ll e^{\delta^{\prime} T} T^{r}=o\left(e^{\delta T}\right)
$$

as $T \rightarrow \infty$. It remains to show that for some $C>0$ independent of $\mathfrak{q}$,

$$
\int_{\mathfrak{q}_{T}} e^{2 \rho(t)} d t \sim_{T \rightarrow \infty} C \cdot T^{(r-1) / 2} e^{\delta T} .
$$


Making a change of variables and decomposing $\mathfrak{q}_{1}$ into slices parallel to the hyperplane $\{2 \rho=0\}$, we have

$$
\int_{\mathfrak{q}_{T}} e^{2 \rho(t)} d t=T^{r} \int_{\mathfrak{q}_{1}} e^{2 T \rho(t)} d t=T^{r} \int_{0}^{\delta} e^{T x} \phi(x) d x,
$$

where $\phi(x)=\operatorname{Vol}_{r-1}\left(\mathfrak{q}_{1} \cap\{2 \rho=x\}\right)$.

First, we show that for some $c_{1}>0$ independent of $\mathfrak{q}$, we have

$$
\phi(x) \sim_{x \rightarrow \delta^{-}} c_{1} \cdot(\delta-x)^{(r-1) / 2} .
$$

We identify $\mathfrak{a}$ with the set $\left\{\left(t_{1}, \ldots, t_{r}\right) \in \mathbb{R}^{r}\right\}$ and denote by $Q$ the positive quadratic form on $\mathfrak{a}$ defined by the norm. After a linear change of variables, we may assume that $2 \rho(t)=t_{r}$ and that

$$
Q\left(t_{1}, \ldots, t_{r}\right)=\sum_{i=1}^{r-1} \alpha_{i}\left(t_{i}-\beta_{i} t_{r}\right)^{2}+\alpha_{r} t_{r}^{2}
$$

for some $\alpha_{i}>0$ and $\beta_{i} \in \mathbb{R}$. It is clear that the maximum of $2 \rho(t)$ on the set $\overline{\mathfrak{a}}_{1}=\{t: Q(t) \leq 1\}$ is achieved when $t_{i}=\beta_{i} t_{r}$ for $i=1, \ldots, r-1$ and $\alpha_{r} t_{r}^{2}=1$. This implies that $\delta=\alpha_{r}^{-1 / 2}$. Since for $x$ close to $\delta$, the set $\mathfrak{q}_{1}^{+} \cap\{2 \rho=x\}$ is defined by the condition $Q\left(t_{1}, \ldots, t_{r-1}, x\right) \leq 1$, we have

$$
\begin{aligned}
\phi(x) & =\operatorname{Vol}_{r-1}\left(\left\{\left(t_{1}, \ldots, t_{r-1}\right): \sum_{i=1}^{r-1} \alpha_{i}\left(t_{i}-\beta_{i} x\right)^{2} \leq 1-\alpha_{r} x^{2}\right\}\right) \\
& =c_{1} \cdot\left(1-\alpha_{r} x^{2}\right)^{(r-1) / 2}=c_{1} \cdot\left(\frac{1-x^{2}}{\delta^{2}}\right)^{(r-1) / 2}
\end{aligned}
$$

for a constant $c_{1}>0$. This proves (5.6). Now, by (5.6) and l'Hôpital's rule,

$$
\beta(x) \stackrel{\text { def }}{=} \int_{0}^{x} \phi(\delta-u) d u \sim_{x \rightarrow 0^{+}} c_{2} \cdot x^{(r+1) / 2}
$$

for some $c_{2}>0$. Thus, by the abelian theorem (see [Wi, Corollary 1.a, page 182]),

$$
\begin{aligned}
\int_{0}^{\delta} e^{T x} \phi(x) d x & =e^{\delta T} \int_{0}^{\delta} e^{-T x} \phi(\delta-x) d x \\
& =e^{\delta T} \int_{0}^{\infty} e^{-T x} d \beta(x) \sim_{T \rightarrow \infty} c_{3} \cdot T^{-(r+1) / 2} e^{\delta T}
\end{aligned}
$$

for some $c_{3}>0$. It is clear that $c_{3}$ is independent of $\mathfrak{q}$. This proves (5.5) and the lemma. 
For $T>0$ and $R \subset G$, define

$$
R_{T}=\{r \in R: d(K, K r)<T\} .
$$

Since $G_{T}=K A_{T}^{+} K$, by combining the previous lemma and (5.3), we deduce the following.

COROLLARY 5.8

For some $C>0$,

$$
\operatorname{Vol}\left(G_{T}\right) \sim_{T \rightarrow \infty} C \cdot T^{(r-1) / 2} e^{\delta T}
$$

In particular, we have the following lemma.

LEMMA 5.9

For some functions $a(\varepsilon)$ and $b(\varepsilon)$ such that $a(\varepsilon) \rightarrow 1$ and $b(\varepsilon) \rightarrow 1$ as $\varepsilon \rightarrow 0^{+}$, we have

$$
a(\varepsilon) \leq \liminf _{T \rightarrow \infty} \frac{\operatorname{Vol}\left(G_{T-\varepsilon}\right)}{\operatorname{Vol}\left(G_{T}\right)} \leq \limsup _{T \rightarrow \infty} \frac{\operatorname{Vol}\left(G_{T+\varepsilon}\right)}{\operatorname{Vol}\left(G_{T}\right)} \leq b(\varepsilon) .
$$

For $T, C>0$, and $R \subset A$, define

$$
\begin{aligned}
R(C) & =\left\{r \in R: \alpha(\log r) \geq C \text { for all } \alpha \in \Phi^{+}\right\}, \\
R_{T}(C) & =R_{T} \cap R(C) .
\end{aligned}
$$

\section{LEMMA 5.11}

Let $Q \subset A^{+}$be a convex cone, centered at the origin, that contains the barycenter in its interior. Then for any fixed $C>0$,

$$
\int_{Q_{T}(C)} \xi(\log a) d a \sim_{T \rightarrow \infty} \int_{A_{T}^{+}} \xi(\log a) d a .
$$

In particular,

$$
\operatorname{Vol}\left(K A_{T}^{+} K\right) \sim_{T \rightarrow \infty} \operatorname{Vol}\left(K A_{T}^{+}(C) K\right)
$$

\section{Proof}

It suffices to prove the lemma when $Q$ is contained in the interior of $A^{+}$. Then there exists $T_{0}>0$ such that $Q_{T}(C) \supset Q_{T}-Q_{T_{0}}$ for all sufficiently large $T>0$. Thus, the lemma follows from Lemma 5.4. 


\section{Equidistribution of solvable flows}

Let $G$ be a connected noncompact semisimple Lie group with finite center which is realized as a closed subgroup of a Lie group $L, \Lambda$ a lattice in $L$, and $Q$ a closed subgroup of $G$ which contains a maximal connected split solvable subgroup of $G$. In this section, we investigate the distribution of orbits of $Q$ in the homogeneous space $\Lambda \backslash L$.

Let $K_{0}$ be a maximal compact subgroup of $G$, and let $A_{0}$ be a split Cartan subgroup of $G$ with respect to $K_{0}$. Then $G=K_{0} A_{0}^{+} K_{0}$ for any positive Weyl chamber $A_{0}^{+}$in $A_{0}$. Denote by $d$ an invariant Riemannian metric on $K_{0} \backslash G$. For $g \in G, R \subset G$, $\Omega \subset K_{0}$, and $T>0$, define

$$
R_{T}(g, \Omega)=\left\{r \in R: d\left(K_{0}, K_{0} g r\right)<T, r \in g^{-1} K_{0} A_{0}^{+} \Omega\right\}
$$

and $R_{T}(g)=R_{T}\left(g, K_{0}\right)$. Note that

$$
R_{T}\left(k_{0} g, \Omega\right)=R_{T}(g, \Omega)=g^{-1}(g R)_{T}(e, \Omega)
$$

for any $k_{0} \in K_{0}$.

The main result in this section is Theorem 6.1 on the equidistribution of the sets $Q_{T}(g, \Omega)$ as $T \rightarrow \infty$. Let $\mu$ be the Haar measure on $L$ so that $\mu(\Lambda \backslash L)=1$, let $v_{0}$ be the probability Haar measure on $K_{0}$, and let $\rho$ be a right-invariant Haar measure on $Q$. Denote by $G_{n}$ the product of all noncompact simple factors of $G$.

\section{THEOREM 6.1}

Suppose that for $y \in \Lambda \backslash L$, the orbit $y G_{n}$ is dense in $\Lambda \backslash L$. Then for $g \in G$, any Borel subset $\Omega \subset K_{0}$ with boundary of measure zero, and $f \in C_{c}(\Lambda \backslash L)$, we have

$$
\lim _{T \rightarrow \infty} \frac{1}{\rho\left(Q_{T}(g)\right)} \int_{Q_{T}(g, \Omega)} f\left(y q^{-1}\right) d \rho(q)=v_{0}\left(M_{0} \Omega\right) \int_{\Lambda \backslash L} f d \mu .
$$

The rest of this section is devoted to a proof of Theorem 6.1. We begin by stating a theorem of Shah. Recall that a sequence $\left\{g_{i}\right\} \subset G$ is called strongly divergent if for every projection $\pi$ from $G$ to its noncompact factor, $\pi\left(g_{i}\right) \rightarrow \infty$ as $i \rightarrow \infty$.

\section{THEOREM 6.2 (Shah [S, Corollary 1.2])}

Suppose that for $y \in \Lambda \backslash L$, the orbit $y G_{n}$ is dense in $\Lambda \backslash L$. Let $\left\{g_{i}\right\} \subset G$ be a strongly divergent sequence. Then for any $f \in C_{c}(\Lambda \backslash L)$ and any Borel subset $U$ in $K_{0}$ with boundary of measure zero,

$$
\lim _{i \rightarrow \infty} \int_{U} f\left(y k g_{i}\right) d v_{0}(k)=v_{0}(U) \int_{\Lambda \backslash L} f d \mu .
$$




\section{Remark 6.3}

Although this theorem was stated in [S] only for the case $U=K_{0}$, its proof works equally well when $U$ is an open subset of $K_{0}$ with boundary of measure zero, and approximating Borel sets by open sets, one can check that Theorem 6.2 holds in the above generality.

Let $K=g^{-1} K_{0} g$, and let $B$ be a maximal connected split solvable subgroup of $G$. By Lemma 4.1, there exists a split Cartan subgroup $A$ with an ordering on the root system $\Phi$ such that $G=K A^{+} K$ and $B=A N$, where $N$ is the subgroup generated by all positive-root subgroups of $A$ in $G$. Let $M$ be the centralizer of $A$ in $K$.

Let $m$ be the Haar measure on $G$ so that (5.1) holds with respect to $K_{0}$ and $A_{0}^{+}$. It follows from the uniqueness of the Haar measure that for every $g \in G$, there exists $c_{g}>0$ such that

$$
\int_{G} f d m=c_{g} \int_{K_{0}} \int_{B} f\left(g^{-1} k g b\right) d \rho(b) d \nu_{0}(k), \quad f \in C_{c}(G) .
$$

In particular,

$$
c_{g} \rho\left(B_{T}(g)\right)=\operatorname{Vol}\left(G_{T}(g)\right)=\operatorname{Vol}\left(G_{T}(e)\right) .
$$

We normalize $\rho$ so that $c_{e}=1$. Let $v$ be the probability Haar measure on $K$.

We use notation from Section 4. In particular, (4.2) holds.

PROPOSITION 6.6

Suppose that for $y \in \Lambda \backslash L$, the orbit $y G_{n}$ is dense in $\Lambda \backslash L$. Let $U=V W$ be an open neighborhood of e in $K$, where $V$ is an open neighborhood of $e$ in $S, W$ is an open neighborhood of e in $M$ such that $\sigma(\partial V)=\tau(\partial W)=0$, and $\Omega$ is a Borel subset of $K_{0}$ such that $v_{0}(\partial \Omega)=0$. Then for any $f \in C_{c}(\Lambda \backslash L)$,

$$
\frac{1}{\rho\left(B_{T}(g)\right)} \int_{U} \int_{B_{T}(g, \Omega)} f\left(y b^{-1} k^{-1}\right) d \rho(b) d k \rightarrow v(U) v_{0}\left(M_{0} \Omega\right) \int_{\Lambda \backslash L} f d \mu
$$

as $T \rightarrow \infty$.

\section{Proof}

Since both $A_{0}$ and $g \mathrm{Ag}^{-1}$ are split Cartan subgroups with respect to $K_{0}$, there exists $k_{0} \in K_{0}$ such that $A_{0}=k_{0} g A g^{-1} k_{0}^{-1}$. Hence, replacing $g$ by $k_{0} g$, we may assume that $A=g^{-1} A_{0} g$.

Let $d$ be the Riemannian metric on $K \backslash G$ induced from the metric on $K_{0} \backslash G$ by the map $K x \mapsto K g x$, and let notation $A_{T}^{+}, A_{T}^{+}(C)$, and $A^{+}(C)$ be defined as in Section 5 . 
For instance,

$$
A_{T}^{+}=\left\{a \in A^{+}: d\left(K_{0} g, K_{0} g a\right)<T\right\} .
$$

Since $K A_{T}^{+} K=g^{-1} G_{T}(e) g$, it follows from (5.3) and (6.5) that for every $T>0$,

$$
\rho\left(B_{T}(e)\right)=\int_{A_{T}^{+}} \xi(\log a) d a,
$$

and by Lemma 5.11,

$$
\int_{A_{T}^{+}-A_{T}^{+}(C)} \xi(\log a) d a=o\left(\rho\left(B_{T}(e)\right)\right) \quad \text { as } T \rightarrow \infty .
$$

Since $B_{T}(g, \Omega)=B_{T}\left(g, M_{0} \Omega\right)$, we may assume without loss of generality that $M_{0} \Omega=\Omega$.

We have

$$
\begin{aligned}
U B_{T}(g, \Omega) & =g^{-1} K_{0} A_{0 T}^{+}(e) \Omega \cap U B=K A_{T}^{+}\left(g^{-1} \Omega g\right) g^{-1} \cap U B \\
& =\left\{k_{1} a k_{2} g^{-1}: k_{1} \in K, a \in A_{T}^{+}, k_{2} \in \Omega_{k_{1}}(a)\right\}
\end{aligned}
$$

where

$$
\Omega_{k_{1}}(a)=\left\{k_{2} \in g^{-1} \Omega g: k_{2} g^{-1} B \in a^{-1} k_{1}^{-1} U B\right\} .
$$

By (5.1) and (6.4),

$$
\begin{aligned}
c_{g} & \int_{U} \int_{B_{T}(g, \Omega)} f\left(y b^{-1} k^{-1}\right) d \rho(b) d \nu(k)=\int_{U B_{T}(g, \Omega)} f\left(y x^{-1}\right) d m(x) \\
= & \int_{k_{1} a k_{2} g^{-1} \in U B_{T}(g, \Omega)} f\left(y g k_{2}^{-1} a^{-1} k_{1}^{-1}\right) \xi(\log a) d \nu\left(k_{2}\right) d a d \nu\left(k_{1}\right) \\
= & \int_{k_{1} \in K} \int_{a \in A_{T}^{+}} \int_{k_{2} \in \Omega_{k_{1}}(a)} f\left(y g k_{2}^{-1} a^{-1} k_{1}^{-1}\right) \xi(\log a) d \nu\left(k_{2}\right) d a d \nu\left(k_{1}\right) .
\end{aligned}
$$

Step 1. We claim that for every $m \in M$,

$$
\begin{aligned}
& \lim _{T \rightarrow \infty} \frac{1}{\rho\left(B_{T}(e)\right)} \int_{V} \int_{A_{T}^{+}} \int_{\Omega_{s m}(a)} f\left(y g k^{-1} a^{-1}(s m)^{-1}\right) \xi(\log a) d \nu(k) d a d \sigma(s) \\
& =\sigma(V) \cdot v\left(g^{-1} \Omega g \cap m^{-1} S W k_{g}^{-1}\right) \cdot \int_{\Lambda \backslash L} f d \mu
\end{aligned}
$$

where $k_{g} \in K$ such that $k_{g} B=g^{-1} B$. 
Let $\Omega_{m}=g^{-1} \Omega g \cap m^{-1} S W k_{g}^{-1}$. One can check that $v\left(\partial \Omega_{m}\right)=0$. To show the claim, we first fix $s \in V$ and $\varepsilon>0$. Note that

$$
\Omega_{s m}(a)=\left\{k \in g^{-1} \Omega g: k k_{g} B \in a^{-1} m^{-1}\left(s^{-1} V\right) W B\right\} .
$$

By Lemma 4.3(1), there exists $C>0$ such that

$$
\int_{\Omega_{m}-\Omega_{s m}(a)}\left|f\left(y g k^{-1} a^{-1}(s m)^{-1}\right)\right| d \nu(k) \leq \varepsilon \quad \text { for all } a \in A_{T}^{+}(C),
$$

and by Theorem 6.2 for any sufficiently large $C>0$ and $a \in A_{T}^{+}(C)$, we have

$$
\left|\int_{\Omega_{m}} f\left(y g k^{-1} a^{-1}(s m)^{-1}\right) d \nu(k)-v\left(\Omega_{m}\right) \int_{\Lambda \backslash L} f d \mu\right|<\varepsilon .
$$

Hence,

$$
\begin{aligned}
& \mid \int_{A_{T}^{+}(C)} \int_{\Omega_{s m}(a)} f\left(y g k^{-1} a^{-1}(s m)^{-1}\right) \xi(\log a) d v(k) d a \\
& \quad-\int_{A_{T}^{+}(C)} \xi(\log a) d a \cdot v\left(\Omega_{m}\right) \cdot \int_{\Lambda \backslash L} f d \mu \mid \\
& \quad \leq \int_{A_{T}^{+}(C)} \int_{\Omega_{m}-\Omega_{s m}(a)}\left|f\left(y g k^{-1} a^{-1}(s m)^{-1}\right)\right| \xi(\log a) d v(k) d a \\
& \quad+\int_{A_{T}^{+}(C)}\left|\int_{\Omega_{m}} f\left(y g k^{-1} a^{-1}(s m)^{-1}\right) d \nu(k)-v\left(\Omega_{m}\right) \int_{\Lambda \backslash L} f d \mu\right| \xi(\log a) d a \\
& \quad \leq 2 \varepsilon \int_{A_{T}^{+}(C)} \xi(\log a) d a \leq 2 \varepsilon \int_{A_{T}^{+}} \xi(\log a) d a .
\end{aligned}
$$

Thus, by (6.7) and (6.8),

$$
\begin{aligned}
& \mid \int_{A_{T}^{+}} \int_{\Omega_{s m}(a)} f\left(y g k^{-1} a^{-1}(s m)^{-1}\right) \xi(\log a) d \nu(k) d a \\
& \quad-\rho\left(B_{T}(e)\right) \nu\left(\Omega_{m}\right) \int_{\Lambda \backslash L} f d \mu \mid \leq 2 \varepsilon \rho\left(B_{T}(e)\right)+o\left(\rho\left(B_{T}(e)\right)\right) .
\end{aligned}
$$

This shows that for every $s \in V$,

$$
\begin{aligned}
& \lim _{T \rightarrow \infty} \frac{1}{\rho\left(B_{T}(e)\right)} \int_{A_{T}^{+}} \int_{\Omega_{s m}(a)} f\left(y g k^{-1} a^{-1}(s m)^{-1}\right) \xi(\log a) d \nu(k) d a \\
& \quad=v\left(\Omega_{m}\right) \int_{\Lambda \backslash L} f d \mu .
\end{aligned}
$$

Therefore, (6.10) follows from the Lebesgue dominated convergence theorem. 
Step 2. We claim that for every $m \in M$, $\lim _{T \rightarrow \infty} \frac{1}{\rho\left(B_{T}(e)\right)} \int_{S-\bar{V}} \int_{A_{T}^{+}} \int_{\Omega_{s m}(a)} f\left(y g k^{-1} a^{-1}(s m)^{-1}\right) \xi(\log a) d \nu(k) d a d \sigma(s)=0$.

Let $s \in S-\bar{V}$, and let $\varepsilon>0$. By Lemma 4.3(2), there exists $C>0$ such that

$$
\nu\left(\Omega_{s m}(a)\right)<\varepsilon \quad \text { for all } a \in A_{T}^{+}(C) .
$$

Hence, by (6.7),

$$
\begin{aligned}
& \frac{1}{\rho\left(B_{T}(e)\right)} \int_{A_{T}^{+}(C)} \int_{\Omega_{s m}(a)}\left|f\left(y g k^{-1} a^{-1}(s m)^{-1}\right)\right| \xi(\log a) d \nu(k) d a \\
& \quad \leq \frac{\varepsilon \cdot \sup |f|}{\rho\left(B_{T}(e)\right)} \int_{A_{T}^{+}(C)} \xi(\log a) d a \leq \varepsilon \cdot \sup |f| .
\end{aligned}
$$

Since $\varepsilon>0$ is arbitrary, it follows from (6.8) that for any $s \in S-\bar{V}$,

$$
\lim _{T \rightarrow \infty} \frac{1}{\rho\left(B_{T}(e)\right)} \int_{A_{T}^{+}} \int_{\Omega_{s m}(a)} f\left(y g k^{-1} a^{-1}(s m)^{-1}\right) \xi(\log a) d \nu(k) d a=0 .
$$

Hence, (6.12) follows from the Lebesgue dominated convergence theorem.

Since $\sigma(\partial V)=0$, by combining (6.10) and (6.12) we deduce that for every $m \in M$,

$$
\begin{aligned}
\lim _{T \rightarrow \infty} & \frac{1}{\rho\left(B_{T}(e)\right)} \int_{S} \int_{A_{T}^{+}} \int_{\Omega_{s m}(a)} f\left(y g k^{-1} a^{-1}(s m)^{-1}\right) \xi(\log a) d \nu(k) d a d \sigma(s) \\
& =\sigma(V) \cdot v\left(g^{-1} \Omega g \cap m^{-1} S W k_{g}^{-1}\right) \cdot \int_{\Lambda \backslash L} f d \mu .
\end{aligned}
$$

Thus, by (4.2), the Lebesgue dominated convergence theorem, and Lemma 4.5,

$$
\begin{aligned}
\lim _{T \rightarrow \infty} & \frac{1}{\rho\left(B_{T}(e)\right)} \int_{K} \int_{A_{T}^{+}} \int_{\Omega_{k_{1}}(a)} f\left(y g k_{2}{ }^{-1} a^{-1}\left(k_{1}\right)^{-1}\right) \xi(\log a) d \nu\left(k_{2}\right) d a d \nu\left(k_{1}\right) \\
\quad & \sigma(V) \cdot \int_{M} \nu\left(g^{-1} \Omega g \cap m^{-1} S W k_{g}^{-1}\right) d \tau(m) \cdot \int_{\Lambda \backslash L} f d \mu \\
& =\sigma(V) \nu\left(g^{-1} \Omega g\right) \tau(W) \int_{\Lambda \backslash L} f d \mu=v(U) \nu_{0}(\Omega) \int_{\Lambda \backslash L} f d \mu
\end{aligned}
$$

Finally, the proposition follows from (6.5) and (6.9). 


\section{Proof of Theorem 6.1}

Let $B$ be a maximal connected split solvable subgroup of $G$ contained in $Q$. Since $G=K B$, we have $Q=D B$ for $D=K \cap Q$. Then

$$
Q_{T}(g, \Omega)=D B_{T}(g, \Omega)
$$

and for suitable Haar measures $\rho_{B}$ and $v_{D}$ on $B$ and $D$, respectively,

$$
\int_{Q} f(q) d \rho(q)=\int_{D} \int_{B} f(d b) d \rho_{B}(b) d v_{D}(d), \quad f \in C_{c}(Q) .
$$

Hence, Theorem 6.1 for $Q$ follows from Theorem 6.1 for $B$ and the Lebesgue dominated convergence theorem. Thus, we may assume that $Q=B$.

Let $\varepsilon>0$. One can find a neighborhood $U$ of $e$ in $K$, as in Proposition 6.6 and functions $f^{+}, f^{-} \in C_{c}(\Lambda \backslash L)$, such that

$$
f^{-}\left(x k^{-1}\right) \leq f(x) \leq f^{+}\left(x k^{-1}\right) \quad \text { for all } x \in \Lambda \backslash L \text { and } k \in U
$$

and

$$
\int_{\Lambda \backslash L}\left|f^{+}-f^{-}\right| d \mu \leq \varepsilon
$$

Put

$$
F_{T}(k)=\frac{1}{\rho\left(B_{T}(g)\right)} \int_{B_{T}(g, \Omega)} f\left(y b^{-1} k^{-1}\right) d \rho(b), \quad k \in K .
$$

For any $k \in U$,

$$
\begin{aligned}
& \frac{1}{\rho\left(B_{T}(g)\right)} \int_{B_{T}(g, \Omega)} f^{-}\left(y b^{-1} k^{-1}\right) d \rho(b) \leq F_{T}(e) \\
& \leq \frac{1}{\rho\left(B_{T}(g)\right)} \int_{B_{T}(g, \Omega)} f^{+}\left(y b^{-1} k^{-1}\right) d \rho(b) .
\end{aligned}
$$

Integrating over $U \subset K$, we obtain

$$
\begin{aligned}
& \frac{1}{\rho\left(B_{T}(g)\right)} \int_{U} \int_{B_{T}(g, \Omega)} f^{-}\left(y b^{-1} k^{-1}\right) d \rho(b) d \nu(k) \leq v(U) \cdot F_{T}(e) \\
& \leq \frac{1}{\rho\left(B_{T}(g)\right)} \int_{U} \int_{B_{T}(g, \Omega)} f^{+}\left(y b^{-1} k^{-1}\right) d \rho(b) d \nu(k) .
\end{aligned}
$$


Hence, by Proposition 6.6,

$$
\begin{aligned}
& v(U) v_{0}\left(M_{0} \Omega\right) \int_{\Lambda \backslash L} f^{-} d \mu \leq v(U) \cdot \liminf _{T \rightarrow \infty} F_{T}(e) \\
& \quad \leq v(U) \cdot \limsup _{T \rightarrow \infty} F_{T}(e) \leq v(U) v_{0}\left(M_{0} \Omega\right) \int_{\Lambda \backslash L} f^{+} d \mu .
\end{aligned}
$$

This shows that

$$
\begin{aligned}
& v_{0}\left(M_{0} \Omega\right) \cdot\left(\int_{\Lambda \backslash L} f d \mu-\varepsilon\right) \leq \liminf _{T \rightarrow \infty} F_{T}(e) \\
& \quad \leq \limsup _{T \rightarrow \infty} F_{T}(e) \leq v_{0}\left(M_{0} \Omega\right) \cdot\left(\int_{\Lambda \backslash L} f d \mu+\varepsilon\right),
\end{aligned}
$$

and the theorem follows.

Since it follows from Shah's result [S, Theorem 1.1] and Ratner's topological rigidity theorem [R2, Theorem 4] that $\overline{y Q}=\overline{y G_{n} Q}$ for every $y \in \Lambda \backslash L$, one may expect that Theorem 6.1 holds under the condition $\overline{y Q}=\Lambda \backslash L$ as well.

\section{LEMMA 6.17}

Let $L$ be a connected semisimple Lie group with finite center, and let $L=L_{c} L_{n}$ be the decomposition of $L$ into the product of compact and noncompact factors. Suppose that for $y \in \Lambda \backslash L$, we have $\overline{y G_{n}} \supset y L_{n}$ and $\overline{y G_{n} Q}=\Lambda \backslash L$. Then the conclusion of Theorem 6.1 holds.

\section{Proof}

Let $B \subset Q$ be a maximal connected split solvable subgroup of $G$. Then $Q=D B$ for $D=K \cap Q$. By Ratner's theorem on orbit closures (see [R2, Theorem 4]), the set $\overline{y G_{n}}$ is a homogeneous space $y G_{0}$ with a probability $G_{0}$-invariant measure $\mu_{0}$, where $G_{0}$ is a closed connected subgroup of $L$ which contains $L_{n}$. Applying Theorem 6.1 to the space $y G_{0}$ and the subgroup $B$, we deduce that

$$
\lim _{T \rightarrow \infty} \frac{1}{\rho_{B}\left(B_{T}(g)\right)} \int_{B_{T}(g, \Omega)} f\left(y b^{-1}\right) d \rho_{B}(b)=v_{0}\left(M_{0} \Omega\right) \int_{y G_{0}} f d \mu_{0}
$$

for every $f \in C_{c}(\Lambda \backslash L)$, where $\rho_{B}$ is a right-invariant Haar measure on $B$. Since $\overline{y G_{n} Q}=\Lambda \backslash L, L_{n} Q=L_{n} D$, and $L_{n} \subset G_{0}$, we have

$$
\Lambda \backslash L=\overline{y G_{n} D}=y G_{0} D .
$$


(Here we used the fact that $D$ is compact.) By (6.13), (6.14), and the Lebesgue dominated convergence theorem,

$$
\lim _{T \rightarrow \infty} \frac{1}{\rho\left(Q_{T}(g)\right)} \int_{Q_{T}(g, \Omega)} f\left(y q^{-1}\right) d \rho(q)=v_{0}\left(M_{0} \Omega\right) \int_{\Lambda \backslash G} f d \tilde{\mu}
$$

for every $f \in C_{c}(\Lambda \backslash L)$, where the measure $\tilde{\mu}$ is defined by

$$
\int_{\Lambda \backslash L} f d \tilde{\mu}=\int_{D} \int_{y G_{0}} f\left(z d^{-1}\right) d \mu_{0}(z) d \nu_{D}(d), \quad f \in C_{c}(\Lambda \backslash L) .
$$

Since $L_{n}$ is a normal subgroup of $L$, it is clear that the measure $\tilde{\mu}$ is invariant under $L_{n} D$. This measure corresponds to a Radon measure $\tilde{\mu}$ on $L$ which is right- $\left(L_{n} D\right)$ invariant and left-Stab ${ }_{L}(y)$-invariant. Namely,

$$
\tilde{\mu}(f)=\int_{\Lambda \backslash L}\left(\sum_{\lambda \in \operatorname{Stab}_{L}(y)} f(\lambda g)\right) d \tilde{\mu}(g), \quad f \in C_{c}(L) .
$$

We have a decomposition $\tilde{\mu}=\int_{L_{c}} \tilde{\mu}_{x} d \omega(x)$ for a Radon measure $\omega$ on $L_{c}$, where $\tilde{\mu}_{x}$ is a right- $L_{n}$-invariant measure on the leaf $x L_{n}$ for $\omega$-a.e. $x \in L_{c}$. Since $L_{n}$ commutes with $x \in L_{c}, \tilde{\mu}_{x}$ is left- $L_{n}$-invariant, too. It follows that the measure $\tilde{\mu}$ is left- $L_{n}$ invariant. Thus, it is left-invariant under $\overline{\operatorname{Stab}_{L}(y) L_{n}} \supset G_{0}$. Setting $E=G_{0} \cap L_{c}$ and $F=D L_{n} \cap L_{c}$, we deduce that the measure $\omega$ is left- $E$-invariant and right$F$-invariant. Note that $E F$ is a closed subset of $L_{c}$, and it follows from (6.18) that $y L_{n} E F=\Lambda \backslash L$. Thus, by the Baire category theorem, the set $E F$ contains an open subset of $L_{c}$. Since the group $E \times F$ acts transitively on $E F$, this implies that $E F$ is open in $L_{c}$. Thus, $L_{c}=E F$ because $L$ is connected. By [K, Theorem 8.32], $\omega$ is a Haar measure on $L_{c}$. This implies that $\tilde{\mu}$ is $L$-invariant, and the lemma follows.

\section{Distribution of lattice points in sectors and the boundary}

In this section, we apply Theorem 6.1 in the case when $\Lambda \backslash L=\Gamma \backslash G$ in order to deduce Theorems 1.1 and 1.2. We use notation from Theorem 1.2. In particular, $A$ is a split Cartan subgroup with respect to $K_{1}, g^{-1} K_{1} g=K_{2}, M_{1}$ is the centralizer of $A$ in $K_{1}$, and $M_{2}=K_{2} \cap Q$.

To simplify notation, we use the following conventions in this section.

- $\quad$ For $R \subset G$ and $T>0$,

$$
R_{T}=\left\{r \in R: d\left(K_{1}, K_{1} g r\right)<T\right\} .
$$

- $\quad$ For $T, C>0, \Omega_{1} \subset K_{1}$, and $\Omega_{2} \subset K_{2}$,

$$
\begin{aligned}
& N_{T}\left(\Omega_{1}, \Omega_{2}\right)=\#\left(\Gamma_{T} \cap g^{-1} K_{1} A^{+} \Omega_{1} \cap \Omega_{2} Q\right), \\
& N_{T}^{C}\left(\Omega_{1}, \Omega_{2}\right)=\#\left(\Gamma_{T} \cap g^{-1} K_{1} A^{+}(C) \Omega_{1} \cap \Omega_{2} Q\right),
\end{aligned}
$$

where $A^{+}(C)$ is defined as in (5.10). 
- $\quad$ For $T, C>0$ and $\Omega \subset K_{1}$,

$$
\begin{aligned}
& Q_{T}(\Omega)=Q_{T} \cap g^{-1} K_{1} A^{+} \Omega, \\
& Q_{T}^{C}(\Omega)=Q_{T} \cap g^{-1} K_{1} A^{+}(C) \Omega .
\end{aligned}
$$

Let $m$ denote the Haar measure on $G$ so that (5.1) holds for $K_{1}$, and let $\rho$ denote the right-invariant Haar measure on $Q$ so that

$$
\int_{G} f d m=\int_{K_{2}} \int_{Q} f(k q) d \rho(q) d \nu_{2}(k), \quad f \in C_{c}(G) .
$$

LEMMA 7.2

For any $C>0, \Omega_{1} \subset K_{1}$, and $\Omega_{2} \subset K_{2}$,

$$
\lim _{T \rightarrow \infty} \frac{1}{m\left(G_{T}\right)}\left(N_{T}\left(\Omega_{1}, \Omega_{2}\right)-N_{T}^{C}\left(\Omega_{1}, \Omega_{2}\right)\right)=0 .
$$

\section{Proof}

Fix $D>C>0$ and $\varepsilon>0$. By Theorem 3.7, there exists a neighborhood $\mathcal{O}$ of $e$ in $G$ such that

$$
\mathcal{O}^{-1} g^{-1} K_{1} A^{+}(D) K_{1} \subset g^{-1} K_{1} A^{+}(C) K_{1} \text {. }
$$

In addition, we may choose $\mathcal{O}$ so that

$$
\Gamma \cap \mathcal{O}^{-1} \mathcal{O}=\{e\} \quad \text { and } \quad \mathcal{O}_{T} \subset G_{T+\varepsilon} \quad \text { for all } T>0 .
$$

It follows from (7.3) that

$$
\mathcal{O} \cdot\left(\Gamma-g^{-1} K_{1} A^{+}(C) K_{1}\right) \subset G-g^{-1} K_{1} A^{+}(D) K_{1} .
$$

Thus,

$$
\begin{aligned}
N_{T} & \left(\Omega_{1}, \Omega_{2}\right)-N_{T}^{C}\left(\Omega_{1}, \Omega_{2}\right) \leq \#\left\{\gamma \in \Gamma_{T}-g^{-1} K_{1} A^{+}(C) K_{1}\right\} \\
& =\frac{1}{m(\mathcal{O})} m\left(\bigcup_{\gamma \in \Gamma_{T}-g^{-1} K_{1} A^{+}(C) K_{1}} \mathcal{O} \gamma\right) \leq \frac{1}{m(\mathcal{O})} m\left(G_{T+\varepsilon}-g^{-1} K_{1} A^{+}(D) K_{1}\right) \\
& =\frac{1}{m(\mathcal{O})} m\left(K_{1}\left(A_{T+\varepsilon}^{+}-A_{T+\varepsilon}^{+}(D)\right) K_{1}\right)=o\left(m\left(G_{T+\varepsilon}\right)\right)
\end{aligned}
$$

by (5.3) and Lemma 5.11. Now, the lemma follows from Lemma 5.9.

The proof of the following lemma is similar and is left to the reader. 


\section{LEMMA 7.4}

For any $C>0$ and $\Omega \subset K_{1}$,

$$
\lim _{T \rightarrow \infty} \frac{1}{m\left(G_{T}\right)}\left(\rho\left(Q_{T}(\Omega)\right)-\rho\left(Q_{T}^{C}(\Omega)\right)\right)=0
$$

\section{Proof of Theorem 1.2}

Without loss of generality, $m(\Gamma \backslash G)=1$. It is easy to check that $\nu_{1}\left(\partial\left(M_{1} \Omega_{1}\right)\right)=$ $\nu_{2}\left(\partial\left(\Omega_{2} M_{2}\right)\right)=0$. Thus, we may assume that $\Omega_{1}=M_{1} \Omega_{1}$ and $\Omega_{2}=\Omega_{2} M_{2}$.

We need to show that

$$
N_{T}\left(\Omega_{1}, \Omega_{2}\right) \sim_{T \rightarrow \infty} v_{1}\left(\Omega_{1}\right) v_{2}\left(\Omega_{2}\right) m\left(G_{T}\right)
$$

Fix any $\varepsilon>0$ and $C>0$. Let $U$ be a neighborhood of $e$ in $K_{1}$ with boundary of measure zero such that $v_{1}\left(\Omega_{1}^{+}-\Omega_{1}^{-}\right)<\varepsilon$, where

$$
\Omega_{1}^{+}=\bigcup_{u \in U} u \Omega_{1} \quad \text { and } \quad \Omega_{1}^{-}=\bigcap_{u \in U} u^{-1} \Omega_{1} \text {. }
$$

One can check that $v_{1}\left(\partial \Omega_{1}^{ \pm}\right)=0$.

By the strong wavefront lemma (Theorem 3.7), there exists a symmetric neighborhood $\mathcal{O}^{\prime}$ of $e$ in $G$ such that

$$
\mathcal{O}^{\prime} g^{-1} K_{1} A^{+}(C) \subset g^{-1} K_{1} A^{+} U \quad \text { and } \quad \mathcal{O}^{\prime} G_{T} \subset G_{T+\varepsilon} \quad \text { for all } T>0 \text {. }
$$

Set $\mathcal{O}=M_{2}\left(\mathcal{O}^{\prime} \cap Q\right) M_{2}$. Note that $\mathcal{O}$ is a symmetric neighborhood of $e$ in $Q$. Using the fact that $M_{2} \subset g^{-1} K_{1} g$, it is easy to check that (7.5) holds for $\mathcal{O}$ as well. We may also assume that $\rho(\partial \mathcal{O})=0$.

Let $f$ be the characteristic function of $\Omega_{2} \Theta \subset G$. Since the decomposition $h=h_{K_{2}} h_{Q}$ for $h_{K_{2}} \in K_{2}$ and $h_{Q} \in Q$ is uniquely determined modulo $M_{2}$ and $\mathrm{M}_{2} \mathcal{O}=\mathcal{O}$, we have

$$
f(h)=\chi_{\Omega_{2}}\left(h_{K_{2}}\right) \chi_{\mathcal{O}}\left(h_{Q}\right)
$$

We also define a function on $\Gamma \backslash G$ by

$$
F(h)=\sum_{\gamma \in \Gamma} f(\gamma h) .
$$

Step 1 . We claim that for any $T>0$,

$$
N_{T}^{C}\left(\Omega_{1}, \Omega_{2}\right) \leq \frac{1}{\rho(\mathcal{O})} \int_{Q_{T+\varepsilon}\left(\Omega_{1}^{+}\right)} F\left(q^{-1}\right) d \rho(q) .
$$


We have

$$
\begin{aligned}
\int_{Q_{T+\varepsilon}\left(\Omega_{1}^{+}\right)} F\left(q^{-1}\right) d \rho(q) & =\int_{Q_{T+\varepsilon}\left(\Omega_{1}^{+}\right)}\left(\sum_{\gamma \in \Gamma} \chi_{\Omega_{2}}\left(\gamma_{K_{2}}\right) \chi_{\mathcal{O}}\left(\gamma_{Q} q^{-1}\right)\right) d \rho(q) \\
& =\sum_{\gamma \in \Gamma: \gamma_{K_{2}} \in \Omega_{2}} \rho\left(Q_{T+\varepsilon}\left(\Omega_{1}^{+}\right) \cap \mathcal{O} \gamma_{Q}\right) .
\end{aligned}
$$

It follows from (7.5) that

$$
\mathcal{O} Q_{T}^{C}\left(\Omega_{1}\right) \subset Q_{T+\varepsilon}\left(\Omega_{1}^{+}\right) .
$$

This implies that for every $\gamma \in \Gamma$ such that $\gamma_{Q} \in Q_{T}^{C}\left(\Omega_{1}\right)$,

$$
\rho\left(Q_{T+\varepsilon}\left(\Omega_{1}^{+}\right) \cap \mathcal{O} \gamma_{Q}\right)=\rho(\mathcal{O}) .
$$

Thus,

$$
\begin{aligned}
\int_{Q_{T+\varepsilon}\left(\Omega_{1}^{+}\right)} F\left(q^{-1}\right) d \rho(q) & \geq \#\left\{\gamma \in \Gamma: \gamma_{K_{2}} \in \Omega_{2}, \gamma_{Q} \in Q_{T}^{C}\left(\Omega_{1}\right)\right\} \cdot \rho(\mathcal{O}) \\
& =N_{T}^{C}\left(\Omega_{1}, \Omega_{2}\right) \rho(\mathcal{O}) .
\end{aligned}
$$

This proves (7.6).

Step 2. We claim that for any $T>0$,

$$
N_{T}\left(\Omega_{1}, \Omega_{2}\right) \geq \frac{1}{\rho(\mathcal{O})} \int_{Q_{T-\varepsilon}^{C}\left(\Omega_{1}^{-}\right)} F\left(q^{-1}\right) d \rho(q) .
$$

As in (7.7),

$$
\int_{Q_{T-\varepsilon}^{C}\left(\Omega_{1}^{-}\right)} F\left(q^{-1}\right) d \rho(q)=\sum_{\gamma \in \Gamma: \gamma_{K_{2}} \in \Omega_{2}} \rho\left(Q_{T-\varepsilon}^{C}\left(\Omega_{1}^{-}\right) \cap \mathcal{O} \gamma_{Q}\right) .
$$

Since $U \Omega_{1}^{-} \subset \Omega_{1}$, we have, by (7.5),

$$
\mathcal{O}^{-1} Q_{T-\varepsilon}^{C}\left(\Omega_{1}^{-}\right) \subset Q_{T}\left(\Omega_{1}\right) .
$$

Therefore, for $\gamma \in \Gamma$ such that $\gamma_{Q} \notin Q_{T}\left(\Omega_{1}\right)$,

$$
\rho\left(Q_{T-\varepsilon}^{C}\left(\Omega_{1}^{-}\right) \cap \mathcal{O} \gamma_{Q}\right)=0 .
$$


By (7.9),

$$
\begin{aligned}
\int_{Q_{T-\varepsilon}^{C}\left(\Omega_{1}^{-}\right)} F\left(q^{-1}\right) d \rho(q) & \leq \#\left\{\gamma \in \Gamma: \gamma_{K_{2}} \in \Omega_{2}, \gamma_{Q} \in Q_{T}\left(\Omega_{1}\right)\right\} \cdot \rho(\mathcal{O}) \\
& =N_{T}\left(\Omega_{1}, \Omega_{2}\right) \rho(\mathcal{O}) .
\end{aligned}
$$

This proves (7.8).

Since the boundary of the set $\Omega_{2} \mathcal{O}$ has measure zero (this can be checked using (7.1)), the function $f$ can be approximated by continuous functions with compact support, and Theorem 6.1 can be applied to the function $F$ (see Lemma 6.17):

$$
\begin{gathered}
\lim _{T \rightarrow \infty} \frac{1}{\rho\left(Q_{T}\right)} \int_{Q_{T}\left(\Omega_{1}^{ \pm}\right)} F\left(q^{-1}\right) d \rho(q)=v_{1}\left(\Omega_{1}^{ \pm}\right) \int_{G / \Gamma} F d m \\
=v_{1}\left(\Omega_{1}^{ \pm}\right) \int_{G} f d m=v_{1}\left(\Omega_{1}^{ \pm}\right) v_{2}\left(\Omega_{2}\right) \rho(\mathcal{O}) .
\end{gathered}
$$

Note that by (7.1), $m\left(G_{T}\right)=\rho\left(Q_{T}\right)$. Combining (7.8), Lemma 7.4, and (7.10), we deduce that

$$
\begin{aligned}
& \liminf _{T \rightarrow \infty} \frac{N_{T}\left(\Omega_{1}, \Omega_{2}\right)}{m\left(G_{T}\right)} \geq \liminf _{T \rightarrow \infty} \frac{1}{m\left(G_{T}\right) \rho(\mathcal{O})} \int_{Q_{T-\varepsilon}^{C}\left(\Omega_{1}^{-}\right)} F\left(q^{-1}\right) d \rho(q) \\
& \quad \geq\left(\liminf _{T \rightarrow \infty} \frac{m\left(G_{T-\varepsilon}\right)}{m\left(G_{T}\right)}\right) \cdot \liminf _{T \rightarrow \infty} \frac{1}{\rho(\mathcal{O}) \rho\left(Q_{T-\varepsilon}\right)} \int_{Q_{T-\varepsilon}\left(\Omega_{1}^{-}\right)} F\left(q^{-1}\right) d \rho(q) \\
& \geq a(\varepsilon) v_{1}\left(\Omega_{1}^{-}\right) v_{2}\left(\Omega_{2}\right) \geq a(\varepsilon)\left(v_{1}\left(\Omega_{1}\right)-\varepsilon\right) v_{2}\left(\Omega_{2}\right),
\end{aligned}
$$

where $a(\varepsilon)$ is defined in Lemma 5.9. Since $\varepsilon>0$ is arbitrary, it follows from Lemma 5.9 that

$$
\liminf _{T \rightarrow \infty} \frac{N_{T}\left(\Omega_{1}, \Omega_{2}\right)}{m\left(G_{T}\right)} \geq v_{1}\left(\Omega_{1}\right) \nu_{2}\left(\Omega_{2}\right) .
$$

By Lemma 7.2, (7.6), and (7.10),

$$
\begin{aligned}
& \limsup _{T \rightarrow \infty} \frac{N_{T}\left(\Omega_{1}, \Omega_{2}\right)}{m\left(G_{T}\right)}=\limsup _{T \rightarrow \infty} \frac{N_{T}^{C}\left(\Omega_{1}, \Omega_{2}\right)}{m\left(G_{T}\right)} \\
& \quad \leq \limsup _{T \rightarrow \infty} \frac{1}{m\left(G_{T}\right) \rho(\mathcal{O})} \int_{Q_{T+\varepsilon}\left(\Omega_{1}^{+}\right)} F\left(q^{-1}\right) d \rho(q) \\
& \quad \leq\left(\limsup _{T \rightarrow \infty} \frac{1}{m\left(G_{T+\varepsilon}\right)}\right) \cdot \limsup _{T \rightarrow \infty} \frac{1}{\rho(\mathcal{O}) \rho\left(Q_{T+\varepsilon}\right)} \int_{Q_{T+\varepsilon}\left(\Omega_{1}^{+}\right)} F\left(q^{-1}\right) d \rho(q) \\
& \quad \leq b(\varepsilon) v_{1}\left(\Omega_{1}^{+}\right) v_{2}\left(\Omega_{2}\right) \leq b(\varepsilon)\left(v_{1}\left(\Omega_{1}\right)+\varepsilon\right) v_{2}\left(\Omega_{2}\right),
\end{aligned}
$$


where $b(\varepsilon)$ is defined in Lemma 5.9. Thus, by Lemma 5.9,

$$
\limsup _{T \rightarrow \infty} \frac{N_{T}\left(\Omega_{1}, \Omega_{2}\right)}{m\left(G_{T}\right)} \leq v_{1}\left(\Omega_{1}\right) v_{2}\left(\Omega_{2}\right)
$$

and the theorem is proved.

\section{Proof of Theorem 1.1}

Note that $G$ is a connected semisimple center-free Lie group with no compact factors and that $K_{x}$ and $K_{y}$ are maximal compact subgroups of $G$. Since $G$ acts transitively on $X$ (see, e.g., [H, Chapter IV, Theorem 3.3]), there exists $g \in G$ such that $y=x g$. Then $K_{y}=g^{-1} K_{x} g$. The closed positive Weyl chamber $\mathscr{W}_{x}$ at $x$ is of the form $x A^{+}$, where $A^{+}$is a positive Weyl chamber in a split Cartan subgroup $A$ of $G$ with respect to $K_{x}$. The stabilizer of $b \in X(\infty)$ in $G$ is a parabolic subgroup $Q$ of $G$ (see [GJT, Chapter III, Proposition 3.8]). In particular, $Q$ contains a maximal connected split solvable subgroup of $G$. Let $\pi: K_{y} \rightarrow b G$ be the map given by $k \mapsto b k^{-1}$. Then for $\Omega_{1} \subset K_{x}$ and $\Omega_{2} \subset b G$, we have

$$
\begin{aligned}
& \#\left\{\gamma \in \Gamma: y \gamma \in \mathscr{W}_{x} \Omega_{1} \cap \mathrm{B}_{\mathrm{T}}(x), b \gamma^{-1} \in \Omega_{2}\right\} \\
& =\#\left\{\gamma \in \Gamma \cap g^{-1} K_{x} A^{+} \Omega_{1} \cap \pi^{-1}\left(\Omega_{2}\right) Q: d\left(K_{x}, K_{x} g \gamma\right)<T\right\} .
\end{aligned}
$$

Note that $\pi$ maps the probability Haar measure on $K_{y}$ to the measure $m_{b, y}$. Using the fact that the map $\pi$ is open, one can check that the set $\pi^{-1}\left(\Omega_{2}\right)$ has boundary of measure zero if $m_{b, y}\left(\partial \Omega_{2}\right)=0$. Hence, Theorem 1.1 follows from Theorem 1.2.

\section{Distribution of lattice points in bisectors}

Let $G$ be a connected semisimple Lie group with finite center, and let $G=K A^{+} K$ be a Cartan decomposition of $G$. To simplify notation, we fix $g \in G$, and for $\Omega_{1}, \Omega_{2} \subset K$ and $T, C>0$, we define

$$
\begin{aligned}
G_{T} & =\{h \in G: d(K, K g h)<T\}, \\
G_{T}\left(\Omega_{1}, \Omega_{2}\right) & =g^{-1} \Omega_{1} A^{+} \Omega_{2} \cap G_{T}, \\
N_{T}\left(\Omega_{1}, \Omega_{2}\right) & =\#\left(\Gamma \cap G_{T}\left(\Omega_{1}, \Omega_{2}\right)\right) .
\end{aligned}
$$

If we set $A_{T}^{+}=\left\{a \in A^{+}: d(K, K a)<T\right\}$, then $G_{T}\left(\Omega_{1}, \Omega_{2}\right)=g^{-1} \Omega_{1} A_{T}^{+} \Omega_{2}$.

Let $G$ be a closed subgroup of a Lie group $L$, and let $\Lambda$ be a lattice in $L$. Let $m$ be a Haar measure on $G$ so that (5.1) holds, and let $\mu$ be the Haar measure on $L$ so that $\mu(\Lambda \backslash L)=1$. 


\section{THEOREM 8.1}

Suppose that for $y \in \Lambda \backslash L$, the orbit $y G_{n}$ is dense in $\Lambda \backslash L$, where $G_{n}$ is the product of all noncompact simple factors of $G$. For any Borel subsets $\Omega_{1}, \Omega_{2} \subset K$ with boundaries of measure zero and $f \in C_{c}(\Lambda \backslash L)$,

$$
\lim _{T \rightarrow \infty} \frac{1}{m\left(G_{T}\right)} \int_{G_{T}\left(\Omega_{1}, \Omega_{2}\right)} f\left(y h^{-1}\right) d m(h)=v\left(\Omega_{1}\right) v\left(\Omega_{2}\right) \int_{\Lambda \backslash L} f d \mu .
$$

\section{Proof}

By (5.1),

$$
\begin{aligned}
\int_{G_{T}\left(\Omega_{1}, \Omega_{2}\right)} f\left(y h^{-1}\right) d m(h) & =\int_{\Omega_{1}} \int_{A_{T}^{+}} \int_{\Omega_{2}} f\left(y k_{2}^{-1} a^{-1} k_{1}^{-1} g\right) \xi(\log a) d k_{2} d a d k_{1} \\
& =\int_{\Omega_{1}} \int_{A_{T}^{+}} \int_{\Omega_{2}^{-1}} f\left(y k_{2} a^{-1} k_{1}^{-1} g\right) \xi(\log a) d k_{2} d a d k_{1}
\end{aligned}
$$

Since $v\left(\Omega_{2}^{-1}\right)=v\left(\Omega_{2}\right)$, by Theorem 6.2 for every $\varepsilon>0$, there exists $C>0$ such that

$$
\left|\int_{\Omega_{2}^{-1}} f\left(y k_{2} a^{-1} k_{1}^{-1} g\right) d k_{2}-v\left(\Omega_{2}\right) \int_{\Lambda \backslash L} f d \mu\right|<\varepsilon
$$

for all $a \in A_{T}^{+}(C)$. Since $m\left(G_{T}\right)=\int_{A_{T}^{+}} \xi(\log a) d a$, it follows from Lemma 5.11 that

$$
\int_{A_{T}^{+}-A_{T}^{+}(C)} \xi(\log a) d a=o\left(m\left(G_{T}\right)\right) \quad \text { as } T \rightarrow \infty .
$$

Combining (8.3) and (8.4), we get

$$
\begin{aligned}
& \left|\int_{A_{T}^{+}} \int_{\Omega_{2}^{-1}} f\left(y k_{2} a^{-1} k_{1}^{-1} g\right) d k_{2} \xi(\log a) d a-v\left(\Omega_{2}\right) \cdot \int_{A_{T}^{+}} \xi(\log a) d a \cdot \int_{\Lambda \backslash L} f d \mu\right| \\
& \quad \leq \int_{A_{T}^{+}(C)}\left|\int_{\Omega_{2}^{-1}} f\left(y k_{2} a^{-1} k_{1}^{-1} g\right) d k_{2}-v\left(\Omega_{2}\right) \int_{\Lambda \backslash L} f d \mu\right| \xi(\log a) d a \\
& \quad+2 \sup |f| \int_{A_{T}^{+}-A_{T}^{+}(C)} \xi(\log a) d a \leq \varepsilon \int_{A_{T}^{+}(C)} \xi(\log a) d a+o\left(m\left(G_{T}\right)\right) \\
& \quad \leq \varepsilon \cdot m\left(G_{T}\right)+o\left(m\left(G_{T}\right)\right) \text { as } T \rightarrow \infty .
\end{aligned}
$$

This proves that for every $k_{1} \in K$,

$$
\lim _{T \rightarrow \infty} \frac{1}{m\left(G_{T}\right)} \int_{A_{T}^{+}} \int_{\Omega_{2}^{-1}} f\left(y k_{2} a^{-1} k_{1}^{-1} g\right) \xi(\log a) d k_{2} d a=v\left(\Omega_{2}\right) \int_{\Lambda \backslash L} f d \mu .
$$


Now, the statement follows from (8.2) and the Lebesgue dominated convergence theorem.

\section{Proof of Theorem 1.6}

We need to show that

$$
N_{T}\left(\Omega_{1} M, M \Omega_{2}\right) \sim_{T \rightarrow \infty} v\left(\Omega_{1} M\right) v\left(M \Omega_{2}\right) \cdot \frac{m\left(G_{T}\right)}{m(\Gamma \backslash G)} .
$$

We may assume without loss of generality that $\Omega_{1} M=\Omega_{1}, M \Omega_{2}=\Omega_{2}$, and $m(\Gamma \backslash G)=1$. Since $M$ contains all compact factors of $G$, we may assume that $G$ contains no compact factors.

Fix $\varepsilon>0$ and $C>0$. There exists a neighborhood $U$ of $e$ in $K$ with boundary of measure zero such that $v\left(\Omega_{i}^{+}-\Omega_{i}^{-}\right)<\varepsilon, i=1,2$, where

$$
\Omega_{i}^{+}=\bigcup_{u \in U} \Omega_{i} u \quad \text { and } \quad \Omega_{i}^{-}=\bigcap_{u \in U} \Omega_{i} u^{-1} .
$$

Note that $v\left(\partial \Omega_{i}^{ \pm}\right)=0, \Omega_{i} U \subset \Omega_{i}^{+}$, and $\Omega_{i}^{-} U \subset \Omega_{i}$. By the strong wavefront lemma (Theorem 3.7), there exists a neighborhood $\mathcal{O}$ of $e$ in $G$ such that

$$
\begin{aligned}
\mathcal{O}^{-1} \Omega_{1} A^{+}(C) \Omega_{2} & \subset \Omega_{1}^{+} A^{+} \Omega_{2}^{+}, \\
\mathcal{O} \Omega_{1}^{-} A^{+}(C) \Omega_{2}^{-} & \subset \Omega_{1} A^{+} \Omega_{2}, \\
\mathcal{O}^{ \pm 1} G_{T} & \subset G_{T+\varepsilon} \text { for all } T>0 .
\end{aligned}
$$

Let

$$
\begin{aligned}
& G_{T}^{C}\left(\Omega_{1}, \Omega_{2}\right)=g^{-1} K A^{+}(C) K \cap G_{T}\left(\Omega_{1}, \Omega_{2}\right), \\
& N_{T}^{C}\left(\Omega_{1}, \Omega_{2}\right)=\#\left(\Gamma \cap G_{T}^{C}\left(\Omega_{1}, \Omega_{2}\right)\right) .
\end{aligned}
$$

It is not hard to show (see Lemmas 7.2, 7.4 for a similar argument) that

$$
\begin{aligned}
m\left(G_{T}\left(\Omega_{1}, \Omega_{2}\right)-G_{T}^{C}\left(\Omega_{1}, \Omega_{2}\right)\right) & =o\left(m\left(G_{T}\right)\right), \\
N_{T}\left(\Omega_{1}, \Omega_{2}\right)-N_{T}^{C}\left(\Omega_{1}, \Omega_{2}\right) & =o\left(m\left(G_{T}\right)\right),
\end{aligned}
$$

as $T \rightarrow \infty$.

Let $f \in C_{c}(G)$ be such that $f \geq 0, \operatorname{supp}(f) \subset \mathcal{O}$, and $\int_{G} f d m=1$. Define a function on $\Gamma \backslash G$ by

$$
F(h)=\sum_{\gamma \in \Gamma} f(\gamma h)
$$


Clearly, $\int_{\Gamma \backslash G} F d m=1$. We claim that

$$
N_{T}^{C}\left(\Omega_{1}, \Omega_{2}\right) \leq \int_{G_{T+\varepsilon}\left(\Omega_{1}^{+}, \Omega_{2}^{+}\right)} F\left(h^{-1}\right) d m(h)
$$

and

$$
N_{T}\left(\Omega_{1}, \Omega_{2}\right) \geq \int_{G_{T-\varepsilon}^{C}\left(\Omega_{1}^{-}, \Omega_{2}^{-}\right)} F\left(h^{-1}\right) d m(h) .
$$

First, we observe that

$$
\int_{G_{T+\varepsilon}\left(\Omega_{1}^{+}, \Omega_{2}^{+}\right)} F\left(h^{-1}\right) d m(h)=\sum_{\gamma \in \Gamma} \int_{G_{T+\varepsilon}\left(\Omega_{1}^{+}, \Omega_{2}^{+}\right) \gamma^{-1}} f\left(h^{-1}\right) d m(h) .
$$

By (8.5),

$$
\mathcal{O}^{-1} G_{T}^{C}\left(\Omega_{1}, \Omega_{2}\right) \subset G_{T+\varepsilon}\left(\Omega_{1}^{+}, \Omega_{2}^{+}\right)
$$

Thus, for every $\gamma \in \Gamma \cap G_{T}^{C}\left(\Omega_{1}, \Omega_{2}\right)$, we have $\mathcal{O}^{-1} \subset G_{T+\varepsilon}\left(\Omega_{1}^{+}, \Omega_{2}^{+}\right) \gamma^{-1}$ and

$$
\int_{G_{T+\varepsilon}\left(\Omega_{1}^{+}, \Omega_{2}^{+}\right) \gamma^{-1}} f\left(h^{-1}\right) d m(h)=1 .
$$

This implies (8.8).

To prove (8.9), we use

$$
\int_{G_{T-\varepsilon}^{C}\left(\Omega_{1}^{-}, \Omega_{2}^{-}\right)} F\left(h^{-1}\right) d m(h)=\sum_{\gamma \in \Gamma} \int_{G_{T-\varepsilon}^{C}\left(\Omega_{1}^{-}, \Omega_{2}^{-}\right) \gamma^{-1}} f\left(h^{-1}\right) d m(h) .
$$

By (8.5),

$$
\mathcal{O} G_{T-\varepsilon}^{C}\left(\Omega_{1}^{-}, \Omega_{2}^{-}\right) \subset G_{T}\left(\Omega_{1}, \Omega_{2}\right)
$$

Therefore, for $\gamma \in \Gamma-G_{T}\left(\Omega_{1}, \Omega_{2}\right)$, we have $\mathcal{O}^{-1} \cap G_{T-\varepsilon}^{C}\left(\Omega_{1}^{-}, \Omega_{2}^{-}\right) \gamma^{-1}=\emptyset$ and

$$
\int_{G_{T-\varepsilon}^{C}\left(\Omega_{1}^{-}, \Omega_{2}\right) \gamma^{-1}} f\left(h^{-1}\right) d m(h)=0 .
$$

This proves (8.9). 
By (8.9), (8.6), and Theorem 8.1,

$$
\begin{aligned}
& \liminf _{T \rightarrow \infty} \frac{N_{T}\left(\Omega_{1}, \Omega_{2}\right)}{m\left(G_{T}\right)} \geq \liminf _{T \rightarrow \infty} \frac{1}{m\left(G_{T}\right)} \int_{G_{T-\varepsilon}^{C}\left(\Omega_{1}^{-}, \Omega_{2}^{-}\right)} F\left(h^{-1}\right) d m(h) \\
& \geq\left(\liminf _{T \rightarrow \infty} \frac{m\left(G_{T-\varepsilon}\right)}{m\left(G_{T}\right)}\right) \cdot \liminf _{T \rightarrow \infty} \frac{1}{m\left(G_{T-\varepsilon}\right)} \int_{G_{T-\varepsilon}^{C}\left(\Omega_{1}^{-}, \Omega_{2}^{-}\right)} F\left(h^{-1}\right) d m(h) \\
& \geq a(\varepsilon) v\left(\Omega_{1}^{-}\right) v\left(\Omega_{2}^{-}\right) \geq a(\varepsilon)\left(v\left(\Omega_{1}\right)-\varepsilon\right)\left(v\left(\Omega_{2}\right)-\varepsilon\right),
\end{aligned}
$$

where $a(\varepsilon)$ is as defined in Lemma 5.9. Since $\varepsilon>0$ is arbitrary, it follows from Lemma 5.9 that

$$
\liminf _{T \rightarrow \infty} \frac{N_{T}\left(\Omega_{1}, \Omega_{2}\right)}{m\left(G_{T}\right)} \geq v\left(\Omega_{1}\right) v\left(\Omega_{2}\right) .
$$

The opposite inequality for lim sup is proved similarly using (8.7), (8.8), and Theorem 8.1 .

\section{Measure-preserving lattice actions}

Let $G$ be a connected semisimple Lie group with finite center, and let $\Gamma_{1}, \Gamma_{2}$ be lattices in $G$. Let $L=G \times G$, let $\Lambda=\Gamma_{1} \times \Gamma_{2}$, let $H=\{(g, g): g \in G\}$, and let $Q$ be a closed subgroup of $H$ containing a maximal connected split solvable subgroup of $H$. Fix an invariant Riemannian metric $d$ on the symmetric space $K \backslash G$, and for $T>0$ and $g \in G$, define

$$
Q_{T}(g)=\{(q, q) \in Q: d(K, K g q)<T\} .
$$

COROLLARY 9.1

Suppose that for $y \in \Lambda \backslash L$, the orbit $y Q$ is dense in $\Lambda \backslash L$. Then for any $f \in C_{c}(\Lambda \backslash L)$,

$$
\lim _{T \rightarrow \infty} \frac{1}{\rho\left(Q_{T}(g)\right)} \int_{Q_{T}(g)} f\left(y q^{-1}\right) d \rho(q)=\int_{\Lambda \backslash L} f d \mu,
$$

where $\rho$ is a right Haar measure on $Q$ and $\mu$ is the L-invariant probability measure on $\Lambda \backslash L$.

\section{Proof}

It suffices to check the conditions of Lemma 6.17. Namely, we show that

$$
\overline{y H_{n}} \supset y L_{n},
$$

where $H_{n}$ and $L_{n}$ denote the product of all noncompact simple factors of $H$ and $L$, respectively. We also denote by $H_{c}$ and $L_{c}$ the product of all compact simple factors of 
$H$ and $L$. By Ratner's theorem on orbit closures (see [R2, Theorem 4]), $\overline{y H_{n}}=y H_{0}$ for some closed subgroup $H_{0}$ of $L$ containing $H_{n}$. Then $y H_{0} H_{c}=\overline{y H}=\Lambda \backslash L$, and it follows from the Baire category theorem (see the proof of Lemma 6.17) that $H_{0} H_{c}$ is an open subset of $L$. Since $H_{0} H_{c}$ is also closed, we conclude that $L=H_{0} H_{c}$. Now, (9.2) follows from Lemma 9.3.

\section{LEMMA 9.3}

Let $S$ be a connected subgroup of $L$ which contains $H_{n}$. Then $S=\left(S \cap L_{n}\right)\left(S \cap L_{c}\right)$.

\section{Proof}

Let $\mathfrak{h}_{n} \subset \mathfrak{s} \subset \mathfrak{l}=\mathfrak{l}_{n} \oplus \mathfrak{l}_{c}$ be the corresponding Lie algebras. We have decompositions

$$
\mathfrak{h}_{n}=\bigoplus_{i} \mathfrak{h}_{n}^{i} \quad \text { and } \quad \mathfrak{l}_{n}=\bigoplus_{i} \mathfrak{l}_{n}^{i}
$$

where $\mathfrak{h}_{n}^{i}$ and $\mathfrak{l}_{n}^{i}$ are the simple ideals of $\mathfrak{h}_{n}$ and $\mathfrak{l}_{n}$, respectively, so that $\mathfrak{h}_{n}^{i} \subset \mathfrak{l}_{n}^{i}$. Note that $\mathfrak{h}_{n}^{i}$ is a maximal subalgebra of $\mathfrak{l}_{n}^{i}$. In particular, $\mathfrak{h}_{n}^{i}$ is its own normalizer in $\mathfrak{l}_{n}^{i}$.

It suffices to show that for every $s=\left(\sum_{i} s_{i}\right)+s_{c} \in \mathfrak{s}$ with $s_{i} \in \mathfrak{l}_{n}^{i}$ and $s_{c} \in \mathfrak{l}_{c}$, we have $s_{i}, s_{c} \in \mathfrak{s}$. Clearly,

$$
\left[\mathfrak{h}_{n}^{i}, s\right]=\left[\mathfrak{h}_{n}^{i}, s_{i}\right] \subset \mathfrak{s} .
$$

If $\left[\mathfrak{h}_{n}^{i}, s_{i}\right]+\mathfrak{h}_{n}^{i} \neq \mathfrak{h}_{n}^{i}$, then $\left[\mathfrak{h}_{n}^{i}, s_{i}\right]+\mathfrak{h}_{n}^{i}$ generates $\mathfrak{l}_{n}^{i}$, and hence, $\mathfrak{l}_{n}^{i} \subset \mathfrak{s}$. Otherwise, $s_{i}$ normalizes $\mathfrak{h}_{n}^{i}$, and it follows that $s_{i} \in \mathfrak{h}_{n}^{i}$. In both cases, $s_{i} \in \mathfrak{s}$. Then $s_{c}=s-\sum_{i} s_{i} \in$ $\mathfrak{s}$, too. This proves the lemma.

Theorem 1.9 can be deduced from Corollary 9.1, as explained in [GW] and [O].

Acknowledgments. Gorodnik thanks the Department of Mathematics at the California Institute of Technology for its hospitality during August 2003, when most of this work was done. We thank François Ledrappier for helpful remarks on the preliminary version of our article.

\section{References}

[DM] S. G. DANI and G. A. MARGULIS, Asymptotic behavior of trajectories of unipotent flows on homogeneous spaces, Proc. Indian Acad. Sci. Math. Sci. 101 (1991), 1-17. MR 1101994492

[DRS] W. DUKE, Z. RUDNICK, and P. SARNAK, Density of integer points on affine homogeneous varieties, Duke Math. J. 71 (1993), 143-179. MR 1230289487 , 488 
[EMM] A. ESKIN, G. MARGULIS, and S. MOZES, Upper bounds and asymptotics in a quantitative version of the Oppenheim conjecture, Ann. of Math. (2) 147 (1998), 93-141. MR 1609447488

[EM] A. ESKIN and C. MCMULLEN, Mixing, counting, and equidistribution in Lie groups, Duke Math. J. 71 (1993), 181 -209. MR 1230290 487, 488, 490, 491, 492

[EMS] A. ESKIN, S. MOZES, and N. SHAH, Unipotent flows and counting lattice points on homogeneous varieties, Ann. of Math. (2) 143 (1996), 253-299. MR 1381987 488

[EO] A. ESKIN and H. OH, Representations of integers by an invariant polynomial and unipotent flows, Duke Math. J. 135 (2006), 481 -506. MR 2272974488

[GO] W. T. GAN and H. OH, Equidistribution of integer points on a family of homogeneous varieties: A problem of Linnik, Compositio Math. 136 (2003), 323 - 352.

MR 1977010488

[G] A. GOOD, Local Analysis of Selberg's Trace Formula, Lecture Notes in Math. 1040, Springer, Berlin, 1983. MR 0727476484

[Go] A. GORODNIK, Lattice action on the boundary of $\mathrm{SL}_{n}(\mathbb{R})$, Ergodic Theory Dynam.

Systems 23 (2003), 1817-1837. MR 2032490488

[GM] A. GORODNIK and F. MAUCOURANT, Proximality and equidistribution on the

Furstenberg boundary, Geom. Dedicata 113 (2005), 197-213. MR 2171305 488

[GOS] A. GORODNIK, H. OH, and N. SHAH, Integral points on symmetric varieties and Satake compactifications, preprint, arXiv:math/0610497v1 [math.NT] 489

[GW] A. GORODNIK and B. WEISS, Distribution of lattice orbits on homogeneous varieties,

Geom. Funct. Anal. 17 (2007), 58-115. 489, 523

[GJT] Y. GUIVARC'H, L. JI, and J. C. TAYLOR, Compactifications of Symmetric Spaces, Progr.

Math. 156, Birkhäuser, Boston, 1998. MR 1633171 487, 518

[H] S. HELGASON, Differential Geometry, Lie Groups, and Symmetric Spaces, Pure Appl.

Math. 80, Academic Press, New York, 1978. MR 0514561 493, 494, 495, 518

[K] A. KNAPP, Representation Theory of Semisimple Groups: An Overview Based on

Examples, Princeton Math. Ser. 36, Princeton Univ. Press, Princeton, 1986.

MR 0855239 493, 513

[Kn] G. KNIEPER, On the asymptotic geometry of nonpositively curved manifolds, Geom.

Funct. Anal. 7 (1997), 755 - 782. MR 1465601503

[L] F. LEDRAPPIER, Ergodic properties of some linear actions, J. Math. Sci. (New York) 105 (2001), 1861 - 1875. MR 1871151488

[M] G. MARGULIS, On Some Aspects of the Theory of Anosov Systems, Springer Monogr.

Math., Springer, Berlin, 2004. MR 2035655 483, 484

[Ma] F. MAUCOURANT, Approximation diophantienne, dynamique des chambres de Weyls et répartition d'orbites de réseaux, Ph.D. dissertation, Université de Lille, Lille, France, 2002. 488

[Mo] G. D. MOSTOW, Strong Rigidity of Locally Symmetric Spaces, Ann. of Math. Stud. 78, Princeton Univ. Press, Princeton, 1973. MR 0385004487

[N] P. NICHOLLS, A lattice point problem in hyperbolic space, Michigan Math. J. 30 (1983), 273-287. MR 0725781484 
[No] A. NOGUEIRA, Orbit distribution on $\mathbb{R}^{2}$ under the natural action of $\mathrm{SL}(2, \mathbb{Z})$, Indag. Math. (N.S.) 13 (2002), 103 - 124. MR 2014978488

[O] H. OH, Lattice action on finite volume homogeneous spaces, J. Korean Math. Soc. 42 (2005), 635-653. MR 2149189 489, 523

[R1] M. RATNER, On Raghunathan's measure conjecture, Ann. of Math. (2) 134 (1991), 545-607. MR 1135878 483, 492

[R2] - Invariant measures and orbit closures for unipotent actions on homogeneous spaces, Geom. Funct. Anal. 4 (1994), 236-257. MR 1262705 488, 489, 512, 523

[S] N. SHAH, Limit distributions of expanding translates of certain orbits on homogeneous spaces, Proc. Indian Acad. Sci. Math. Sci. 106 (1996), 105-125. MR 1403756 483, 488, 492, 506, 507, 512

[Sh] R. SHARP, Sector estimates for Kleinian groups, Port. Math. (N.S.) 58 (2001), 461-471. MR 1881873484

[V] V. VATSAL, Uniform distribution of Heegner points, Invent. Math. 148 (2002), 1 - 46. MR 1892842489

[W] G. WARNER, Harmonic Analysis on Semi-Simple Lie Groups, I, Grundlehren Math. Wiss. 188, Springer, New York, 1972. MR 0498999500

[Wi] D. V. WIDDER, The Laplace Transform, Princeton Math. Ser. 6, Princeton Univ. Press, Princeton, 1941. MR 0005923504

[Wit] D. WITTE [D. W. MORRIS], "Cocompact subgroups of semisimple Lie groups" in Lie Algebra and Related Topics (Madison, Wisc., 1988), Contemp. Math. 110, Amer. Math. Soc., Providence, 1990, 309-313. MR 1079114488

[Z] R. J. ZIMMER, Ergodic Theory and Semisimple Groups, Monogr. Math. 81, Birkhäuser, Basel, 1984. MR 0776417501

\section{Gorodnik}

Department of Mathematics, California Institute of Technology, Pasadena, California 91125, USA; gorodnik@caltech.edu

Oh

Department of Mathematics, Brown University, Providence, Rhode Island 02912, USA; heeoh@math.brown.edu 